\title{
Cobordism, Relative Indices and Stein Fillings
}

\author{
Charles L. Epstein* \\ Department of Mathematics \\ University of Pennsylvania
}

Draft: May 8, 2007

Dedicated with gratitude and admiration to Gennadi Henkin
on the occasion of his 65th birthday.

\begin{abstract}
In this paper we build on the framework developed in [7, 8, 9, to obtain a more complete understanding of the gluing properties for indices of boundary value problems for the $\operatorname{Spin}_{\mathbb{C}}$-Dirac operator with sub-elliptic boundary conditions. We extend our analytic results for sub-elliptic boundary value problems for the $\operatorname{Spin}_{\mathbb{C}}$-Dirac operator, and gluing results for the indices of these boundary problems to $\operatorname{Spin}_{\mathbb{C}^{-}}$ manifolds with several pseudoconvex (pseudoconcave) boundary components. These results are applied to study Stein fillability for compact, 3-dimensional, contact manifolds.
\end{abstract}

\section{Introduction}

In several earlier papers we analyzed Fredholm boundary value problems for the $\operatorname{Spin}_{\mathbb{C}}$-Dirac operator defined by modifying the $\bar{\partial}$-Neumann boundary condition. To apply this analysis we require a compact, $2 n$-dimensional, $\operatorname{Spin}_{\mathbb{C}^{-m a n i f o l d}} X$, with contact boundary, $Y$. The $\operatorname{Spin}_{\mathbb{C}^{-}}$-structure must be

\footnotetext{
${ }^{*}$ Keywords: $\operatorname{Spin}_{\mathbb{C}}$ Dirac operator, index formula, subelliptic boundary value problem, modified $\bar{\partial}$-Neumann condition, almost complex manifolds, contact manifold, relative index conjecture, Bojarski's theorem, tame Fredholm pairs. E-mail: cle@math.upenn.edu This material is based upon work supported by the National Science Foundation under Grant No. 0603973, and the Francis J. Carey term chair. Any opinions, findings, and conclusions or recommendations expressed in this material are those of the author and do not necessarily reflect the views of the National Science Foundation.
} 
defined in a neighborhood, $U$, of $b X$ by an almost complex structure, $J$, see [15]. The contact structure on $Y$ is assumed to be compatible with the almost complex structure in a sense explained below. In our earlier work we assume that the boundary $Y$ is a connected manifold. In this paper we extend the analytic results for sub-elliptic boundary value problems to manifolds with several boundary components, some pseudoconvex and some pseudoconcave. These results are then applied the prove various extensions, to the sub-elliptic case, of Bojarski's gluing formulæ for indices of Dirac operators. Finally these results are applied, along with the classical excision theorem for indices of Gromov and Lawson, to study the set of embeddable structures on a 3d-CR manifold.

The almost complex structure, $J$, defines a splitting of $T X \otimes \mathbb{C} \uparrow_{U}$ into complementary subbundles

$$
T X \otimes \mathbb{C} \uparrow_{U}=T^{1,0} X \oplus T^{0,1} X,
$$

the dual splitting of $T^{*} X \otimes \mathbb{C}$ is denoted by $\Lambda^{1,0} X \oplus \Lambda^{0,1} X$. Though these bundles are only defined in the subset of $X$ where $J$ is defined; to avoid introducing excessive notation, we denote them by $\Lambda^{1,0} X$, etc. This splitting leads to the definition of the $\bar{\partial}$-operator:

$$
\bar{\partial} f=d f \uparrow_{T^{0,1} X}
$$

$\bar{\partial} f$ is a section of $\Lambda^{0,1} X$.

For each $0 \leq p, q \leq n$, we let $\Lambda^{p, q}$ denote the bundle of forms of type $(p, q)$ defined by the almost complex structure. If $\$$ denotes the bundle of complex spinors over $X$, then over $U$ we have the identification:

$$
\$ \uparrow_{U}=\bigoplus_{q=0}^{n} \Lambda^{0, q} X \uparrow_{U}
$$

For each $q$, the $\bar{\partial}$-operator extends to define a map

$$
\bar{\partial}: \mathcal{C}^{\infty}\left(U ; \Lambda^{p, q} X\right) \longrightarrow \mathcal{C}^{\infty}\left(U ; \Lambda^{p, q+1} X\right) .
$$

We select an Hermitian metric $g$ on $T^{1,0} X$, this defines a formal adjoint $\bar{\partial}^{*}$.

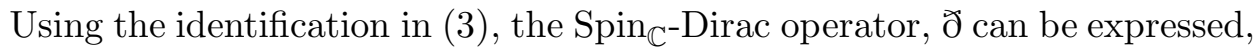
over $U$, as

$$
\check{\partial}=\bar{\partial}+\bar{\partial}^{*}+\mathcal{E}
$$

where $\mathcal{E}: \not \$ \rightarrow \$$ is a bundle endomorphism. 
In this paper, we generally regard manifolds with boundary as closed, so that, for example, $\rho \in \mathcal{C}^{\infty}(X)$ means that $\rho$ is smooth up to, and including the boundary. The notation $\bar{X}$ is used to denote the oriented manifold $X$ with its orientation reversed.

Let $\rho \in \mathcal{C}^{\infty}(X)$ be a defining function for $b X: X=\{x \in X: \rho(x)<0\}$, $d \rho$ is non-vanishing along $b X$. The Hermitian metric on $T^{1,0} X$ defines the interior product operation

$$
\bar{\partial} \rho\rfloor: \Lambda^{p, q} X \longrightarrow \Lambda^{p, q-1} X .
$$

The classical $\bar{\partial}$-Neumann condition for sections $\sigma^{p, q} \in \mathcal{C}^{\infty}\left(X ; \Lambda^{p, q} X\right)$, is the requirement that

$$
\bar{\partial} \rho\rfloor \sigma^{p, q} \uparrow_{b X}=0 .
$$

The boundary of $X$ is assumed to be a contact manifold. The contact structure is compatible with $J$ in that the hyperplane field $H$ on $Y$ is the null-space of the real 1 -form

$$
\theta=i \bar{\partial} \rho\left\lceil_{T Y}\right.
$$

In order for our analytic results to apply, the boundary of $X$ must satisfy one of several convexity properties, which are described by the signature of the Levi-form,

$$
\mathcal{L}_{y}(X, Y)=\frac{1}{2}\left[d \theta_{y}(X, J Y)+d \theta_{y}(Y, J X)\right], \text { for } X \in H_{y} .
$$

A boundary point $y$ is strictly pseudoconvex if $\mathcal{L}_{y}$ is positive definite, and strictly pseudoconcave if $\mathcal{L}_{y}$ is negative definite. Let $Y_{j}$ be a connected component of $Y$; if $\mathcal{L}_{y}>0\left(\mathcal{L}_{y}<0\right)$ for all $y \in Y_{j}$ then we say that $Y_{j}$ is strictly pseudoconvex (pseudoconcave). In our earlier papers we showed how to modify the $\bar{\partial}$-Neumann condition to obtain a sub-elliptic boundary condition provided that each boundary component of $X$ is either strictly pseudoconvex or strictly pseudoconcave. In fact our approach applies so long as $\mathcal{L}_{y}$ is non-degenerate at every boundary point. The modifications to the $\bar{\partial}$-Neumann condition, needed to define a sub-elliptic boundary value problem, depend on the signature of $\mathcal{L}$. In this paper we again focus on boundaries that are either pseudoconvex or pseudoconcave.

In the integrable, strictly pseudoconvex case the reason that the $\bar{\partial}$ Neumann condition itself does not define a Fredholm operator for $\partial$ is that $\bar{\partial}$ has an infinite dimensional null-space in degree 0 , i.e. the holomorphic functions. The reason is simply that $\bar{\partial} \rho\rfloor \sigma^{0,0} \uparrow_{b X}=0$ is always satisfied for 
a $(0,0)$-form. To correct this we need to change the boundary condition in degree 0 . In the classical case there is an orthogonal projector, $\mathcal{S}$ defined on $\mathcal{C}^{\infty}(b X)$, whose range consists of the boundary values of holomorphic functions; it is called "the" Szego" projector. We distinguish this case, by calling this a classical Szegö projector.

The boundary condition is modified in degree zero by requiring

$$
\mathcal{S}\left(\sigma^{0,0} \uparrow_{b X}\right)=0 .
$$

To get a formally self adjoint operator, the boundary condition in degree 1 must also be modified by requiring

$$
\left.(\operatorname{Id}-\mathcal{S})[\bar{\partial} \rho\rfloor \sigma^{0,1} \uparrow_{b X}\right]=0 .
$$

These conditions, along with the $\bar{\partial}$-Neumann condition in degrees greater than 1 , define a projector, $\mathcal{R}_{+}$, acting of sections of $\$^{\dagger} \uparrow_{b X}$. The modified $\bar{\partial}_{-}$ Neumann condition for $\precsim$ on a strictly pseudoconvex manifold is requirement that

$$
\mathcal{R}_{+}\left[\sigma \uparrow_{b X}\right]=0
$$

The pair $(ð, \mathcal{R})$ denotes the operator defined by $ð$ acting on a domain defined by the condition in (12). In our earlier papers we showed that this operator is essentially self adjoint, and it graph closure is a Fredholm operator. The spin-bundles and operators split into even and odd parts. The index of the even part $\left(\check{\partial}^{\mathrm{e}}, \mathcal{R}^{\mathrm{e}}\right)$, computes the renormalized holomorphic Euler characteristic of $X$ :

$$
\operatorname{Ind}\left(\check{\beth}^{\mathrm{e}}, \mathcal{R}^{\mathrm{e}}\right)=\sum_{q=1}^{n}(-1)^{q} \operatorname{dim} H^{0, q}(X) .
$$

The analytic results are generalized to the non-integrable case by introducing the notion of a generalized Szegö projector. This idea appears in [12] and is closely related to that introduced in the appendix to [3]. Briefly, the contact structure on $Y$ defines an algebra of pseudodifferential operators, $\Psi_{H}^{*}(Y)$, called the Heisenberg algebra, see [1, 19]. The classical Szegö projector, $\mathcal{S}$, is an element of $\Psi_{H}^{0}(Y)$. The principal Heisenberg-symbol of $\mathcal{S}$ is defined by the complex structure induced on the fibers of $H$. Generally, if $(Y, H)$ is a contact manifold, then an almost complex structure, $J$, on the fibers of $H$, is positive if the induced Levi-form is positive definite. This data defines a function, $s_{J}$, on $T^{*} Y$, which is, in turn, the principal symbol of an operator $\mathcal{S} \in \Psi_{H}^{0}(Y)$. 
Definition 1. An operator $\mathcal{S} \in \Psi_{H}^{0}(Y)$ is a generalized Szegő projector if

1. $\mathcal{S}^{2}=\mathcal{S}$ and $\mathcal{S}^{*}=\mathcal{S}$.

2. There is a positive almost complex structure $J$ on $H$ so that the principal symbol of $\mathcal{S}$ satisfies:

$$
\sigma_{0}^{H}(\mathcal{S})=s_{J}
$$

Classical Szegö projectors, defined in the integrable case, are generalized Szegö projectors, but more importantly, generalized Szegö projectors exist on any contact manifold with positive almost complex structures. A fundamental fact about generalized Szegö projectors is that if $\mathcal{S}_{1}$ and $\mathcal{S}_{2}$ are two generalized Szegö projectors on $(Y, H)$, then the restriction

$$
\mathcal{S}_{1}: \text { range } \mathcal{S}_{2} \longrightarrow \text { range } \mathcal{S}_{1}
$$

is a Fredholm operator, see [12]. We denote its index by $\operatorname{R}-\operatorname{Ind}\left(\mathcal{S}_{2}, \mathcal{S}_{1}\right)$. A generalized Szegó projector is not determined by its full symbol, indeed, amongst pairs $\left(\mathcal{S}_{1}, \mathcal{S}_{2}\right)$, such that $\mathcal{S}_{1}-\mathcal{S}_{2}$ are smoothing operators, the relative index R-Ind $\left(\mathcal{S}_{2}, \mathcal{S}_{1}\right)$ assumes all integral values.

Using generalized Szegő projectors, the modified pseudoconvex $\bar{\partial}$-condition can be defined on any strictly pseudoconvex $\operatorname{Spin}_{\mathbb{C}}$-manifold, $X$, satisfying the conditions described above. Let $(Y, H)$ be the boundary of $X$, which we suppose is strictly pseudoconvex, and let $\mathcal{S} \in \Psi_{H}^{0}(Y)$, be a generalized Szegó projector. Using the identification in (3), the modified pseudoconvex $\bar{\partial}$-Neumann condition defined by $\mathcal{S}$ is given by

$$
\begin{aligned}
& \mathcal{S}\left[\sigma^{0,0} \uparrow_{b X}\right]=0 \\
& \left.(\operatorname{Id}-\mathcal{S})[\bar{\partial} \rho\rfloor \sigma^{0,1} \uparrow_{b X}\right]=0 \\
& \left.[\bar{\partial} \rho\rfloor \sigma^{0, q}\right]\left\lceil_{b X}=0 \text { for } q \geq 2 .\right.
\end{aligned}
$$

As before these conditions are define by a projector, $\mathcal{R}_{+}$acting on $\mathcal{C}^{\infty}\left(Y ; \$^{\prime} \uparrow_{b X}\right.$ ) .

Definition 2. Let $X$ be a manifold with boundary, $E, F$ two smooth vector bundles over $X$, and $P: \mathcal{C}^{\infty}(X ; E) \rightarrow \mathcal{C}^{\infty}(X ; F)$ a first order differential operator. If $B$ is a pseudodifferential operator acting on sections of $E \uparrow_{b X}$, then $(P, B)$ denotes the differential operator with domain $s \in \mathcal{C}^{\infty}(X ; E)$ satisfying $B\left[s \uparrow_{b X}\right]=0$. 
In [9] it is shown that if $X$ is strictly pseudoconvex, then $\left(ð, \mathcal{R}_{+}\right)$is an essentially self adjoint operator and its graph closure is a Fredholm operator. If $\left(\mathcal{J}^{\text {eo }}, \mathcal{R}_{+}^{\text {eo }}\right)$ are the even and odd parts, then it is also shown that the adjoints satisfy

$$
\left(\check{\partial}^{\mathrm{eo}}, \mathcal{R}_{+}^{\mathrm{eo}}\right)^{*}=\overline{\left(\varlimsup^{\mathrm{oe}}, \mathcal{R}_{+}^{\mathrm{oe}}\right)} .
$$

Below we show that if $X$ is strictly pseudoconcave, then the same results hold with $\mathcal{R}_{+}$replaced by $\mathrm{Id}-\mathcal{R}_{+}$.

In our earlier papers extensive usage is made of gluing constructions, and various formulæ are proved relating the indices of sub-elliptic boundary value problems on the pieces to the index of $\check{\partial}^{e}$ on a boundary-less glued space. In the first part of this paper we extend these results to more general situations allowing multiple boundary components, and a glued space with boundary components. These results are extensions of results of Bojarski in the elliptic case to the sub-elliptic case. As part of this analysis, we consider the structure of the Calderon projector on a $\operatorname{Spin}_{\mathbb{C}}$-manifold with several boundary components.

In the second part of the paper we apply these results to study the problem of embeddability (or Stein fillability) for CR-structures on compact 3-manifolds. Let $X_{+}$be a strictly pseudoconvex surface with boundary the CR-manifold $\left(Y, T_{b}^{0,1} Y\right)$. We suppose that $\left(Y, T_{b}^{0,1}\right)$ is also the boundary of a strictly pseudoconcave manifold $X_{-}$, which contains a positive, compact holomorphic curve, $Z$. Our main result is

Theorem 1. Let $\left(Y, T_{b}^{0,1} Y\right)$ satisfy the conditions above, and let $\mathcal{S}_{0}$ denote the classical Szego" projector defined by the CR-structure on $Y$. If

$$
H_{c}^{2}\left(X_{-} ; \Theta\right)=0 \text { and } \operatorname{deg} N Z \geq 2 g-1,
$$

where $g$ is the genus of $Z$, then there is a constant $M$, such that for a sufficiently small embeddable deformations of the CR-structure, with Szegö projector $\mathcal{S}_{1}$, the relative index satisfies:

$$
\left|\operatorname{R-} \operatorname{Ind}\left(\mathcal{S}_{0}, \mathcal{S}_{1}\right)\right| \leq M \text {. }
$$

As a corollary of this result we conclude that the set of small embeddable deformations of $\left(Y, T_{b}^{0,1} Y\right)$ is closed in the $\mathcal{C}^{\infty}$-topology. This theorem is a considerable generalization of the seminal result of Lempert treating domains in $\mathbb{C}^{2}$, see [17]. It represents the culmination of the line of research begun in [11, 6]. It is proved by combining the index formula from [9] with the Gromov-Lawson excision theorem, 14], and results of Stipsicz on the topology of Stein fillings of circle bundles over Riemann surfaces, see [18]. 


\section{$1 \quad \operatorname{Spin}_{\mathbb{C}}$-boundaries}

Let $X$ be a $2 n$-dimensional $\operatorname{Spin}_{\mathbb{C}}$-manifold with compatible metric $g$. The

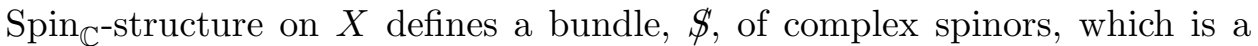
Clifford module for the complexified Clifford bundle of $T^{*} X$. If $d V$ is volume form, then the Clifford action of $i^{n} \boldsymbol{c}(d V)$ splits $\$$ into two subbundles

$$
\$=\phi^{\mathfrak{e}} \oplus \phi^{\pitchfork}
$$

The Clifford action of $\eta_{x} \in T_{x}^{*} X$, a non-vanishing 1-form at $x$, defines isomorphisms:

$$
\boldsymbol{c}\left(\eta_{x}\right): \$_{x}^{\mathrm{eeo}} \longrightarrow \$_{x}^{\mathrm{oe}}
$$

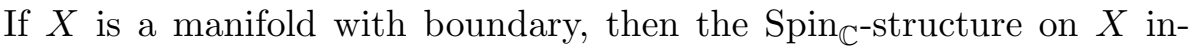

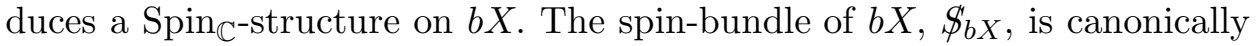
isomorphic to $\$^{\mathrm{e}} \uparrow_{b X}$. Let $t$ be a defining function for $b X$, such that $t<0$ on $X,\|d t\|_{g}=1$, and $\operatorname{grad}_{g} t$ is orthogonal to $T b X \subset T X \uparrow_{b X}$. Under this identification, the Clifford action of $\eta \in T_{x}^{*} b X$, on $\$_{b X}$ is given by

$$
\boldsymbol{c}_{b X}(\eta) \cdot s=\boldsymbol{c}_{X}(-d t) \boldsymbol{c}_{X}(\widetilde{\eta}) \cdot s .
$$

Here $\widetilde{\eta}$ is the extension of $\eta$ to $T_{x} X$ by zero on the orthogonal complement of $T_{x} b X \subset T_{x} X$.

Definition 3. Let $\left(Y, g_{Y}\right)$ be an odd-dimensional $\operatorname{Spin}_{\mathbb{C}^{-} \text {-manifold, such that }}$ there is an even dimensional $\operatorname{Spin}_{\mathbb{C}}$-manifold, $\left(X, g_{X}\right)$ with oriented boundary $Y$. Suppose that $g_{X} \uparrow T Y=g_{Y}$, and the $\operatorname{Spin}_{\mathbb{C}^{-} \text {-structure on } Y \text { satisfies }}$

$$
\$_{Y} \simeq \$_{X}^{\mathrm{e}} \uparrow_{b X}
$$

and, under this identification, the Clifford action of $T^{*} Y$ on $\$_{Y}$ satisfies (22), with $b X=Y$. In this case we say that $\left(Y, \$_{Y}\right)$ is the $\operatorname{Spin}_{\mathbb{C}}$-boundary of $\left(X, \$_{X}\right)$.

In this connection we often consider the boundary with its orientation reversed, $\bar{Y}$. Identifying $\$_{Y}$ with $\$_{X}^{\circ}$ and defining the Clifford action by

$$
\boldsymbol{c}_{b X}(\eta) \cdot s=\boldsymbol{c}_{X}(d t) \boldsymbol{c}_{X}(\widetilde{\eta}) \cdot s,
$$

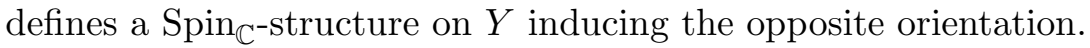

We briefly review the construction of an "invertible double," given in [2]. Let $X$ be a $\operatorname{Spin}_{\mathbb{C}}$-manifold with boundary $Y$, connected or not. The tubular neighborhood theorem implies that there is a neighborhood, $U$, of the 
boundary that is diffeomorphic to $Y \times[-1,0]$. Using this identification, we define the double of $X$ to be the oriented manifold

$$
\widehat{X}=X \amalg_{b X} \bar{X} .
$$

Here $\bar{X}$ denote $X$ with the orientation reversed. The boundary, $Y \times\{0\}$, is now a separating hypersurface in $\widehat{X}$ with neighborhood $V \simeq Y \times(-1,1)$. A function on $\widehat{X}$ is smooth near to $Y$ if smooth with respect to this product structure. The tubular neighborhood theorem implicitly defines a function, $t$ in $V$, taking values in $[-1,1]$. We denote the component of $\widehat{X} \backslash Y \times\{0\}$ where $t<0$ by $X_{+}$and the other component by $X_{-}$.

We can deform the metric in the collar so that

$$
g \uparrow_{V}=d t^{2}+g_{Y},
$$

where $g_{Y}$ is a metric on $Y$. In this case $d t$ is an outward pointing, unit co-vector and $\boldsymbol{c}(-d t)$, Clifford multiplication by $-d t$ defines unitary isomorphisms of the spin-bundles

$$
\boldsymbol{c}(-d t): \phi^{\Uparrow} \uparrow_{V} \rightarrow \phi^{\Uparrow} \uparrow_{V}
$$

The spin-bundle $\widehat{\phi} \rightarrow \widehat{X}$ is defined by using this identification to glue $\phi^{\text {eo }} \uparrow_{V}$ to $\$^{\text {oe }} \uparrow_{V}$. In 2 it is shown that the Dirac operator extends to act on sections of $\widehat{\phi}$, with trivial kernel and co-kernel. Hence $\left(\widehat{X}, \widehat{\Phi}^{\prime}\right)$ is called an invertible double.

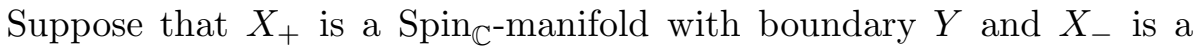
$\operatorname{Spin}_{\mathbb{C}}$-manifold with boundary $\bar{Y}$. Suppose further that the $\operatorname{Spin}_{\mathbb{C}^{-} \text {-structures }}$ on $b X_{ \pm}$are (after a change of orientation on one) isotopic. After attaching cylinders, diffeomorphic to $Y \times[0,1]$, to $X_{+}, X_{-}$, an obvious modification of the invertible double construction from [2] provides a $\operatorname{Spin}_{\mathbb{C}}$-manifold

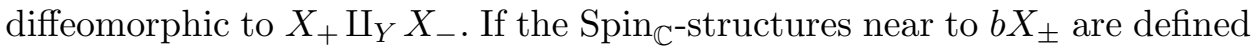
by almost complex structures, then Lemma 8 of [9] shows that this remains true in the added cylinders. The original manifolds $X_{+}, X_{-}$are $\operatorname{Spin}_{\mathbb{C}^{-}}$ isomorphic to open subsets of the glued space. In the sequel it should be understood that $X_{+} \amalg_{Y} X_{-}$refers to the $\operatorname{Spin}_{\mathbb{C}^{-}}$-manifold obtained by such an augmentation and gluing process. In circumstances where there are several manifolds, we use the notation $\partial_{X}$ to indicate the $\operatorname{Spin}_{\mathbb{C}}$-Dirac operator on the manifold $X$.

In this paper we usually assume that $X$ is a $\operatorname{Spin}_{\mathbb{C}}$-manifold with bound-

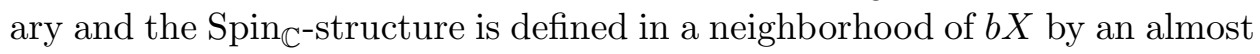
complex structure. In this circumstance the almost complex structure defines a hyperplane field on $b X$, as the ker $\theta$, defined in (8). We usually assume 
that this hyperplane field is a contact structure, and that, as boundaries of $X$, each boundary component is either strictly pseudoconvex or strictly pseudoconcave. For the sake of brevity, in the sequel we describe this cir-

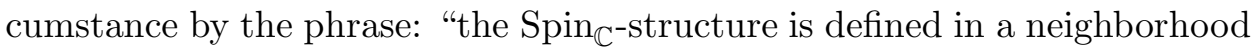
of $b X$ by an almost complex structure, making the components of $b X$ either strictly pseudoconvex or strictly pseudoconcave."

\section{The Calderon projector}

In our earlier papers we showed that the index of a sub-elliptic boundary

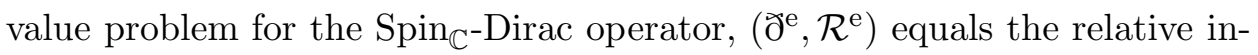
dex between the projector defining the boundary condition, $\mathcal{R}^{\mathrm{e}}$ and the Calderon projector, $\mathcal{P}^{\mathrm{e}}$ :

$$
\operatorname{Ind}\left(\check{\partial}^{\mathrm{e}}, \mathcal{R}^{\mathrm{e}}\right)=\mathrm{R}-\operatorname{Ind}\left(\mathcal{P}^{\mathrm{e}}, \mathcal{R}^{\mathrm{e}}\right) .
$$

This relative index can in turn be computed as a difference of traces, which provides an incisive analytic tool for studying the properties of these indices under deformation and gluing. In this paper we consider manifolds with several boundary components. Many of the analytic results in 2] and our earlier papers are essentially microlocal and so the number of boundary components is of no import. To analyze the behavior of the indices of boundary value problems under gluing it is useful to have more detailed information about the Calderon projector.

Suppose for example that $X$ is a manifold with two boundary components $Y_{0}, Y_{1}$. The Calderon projector acts on sections of

$$
\not \uparrow_{b X} \simeq \not \uparrow_{Y_{0}} \oplus \$ \uparrow_{Y_{1}} \simeq \$_{0} \oplus \$_{1}
$$

We can use this splitting to write the Calderon projector in block form

$$
\mathcal{P}=\left(\begin{array}{ll}
\mathcal{P}_{00} & \mathcal{P}_{01} \\
\mathcal{P}_{10} & \mathcal{P}_{11}
\end{array}\right)
$$

The principal symbols of the diagonal terms are projectors, the off-diagonal terms are smoothing operators. It is of interest to when when this projector can be deformed, through projectors, to a diagonal matrix. A simple analytic sufficient condition is that $\mathcal{P}_{00}$ and $\mathcal{P}_{11}$ are projectors.

Proposition 1. Suppose that $\mathcal{P}$ is a projector with block form as in (30). If $\mathcal{P}_{00}^{2}=\mathcal{P}_{00}$ and $\mathcal{P}_{11}^{2}=\mathcal{P}_{11}$, then

$$
\mathcal{P}_{t, s}=\left(\begin{array}{cc}
\mathcal{P}_{00} & t \mathcal{P}_{01} \\
s \mathcal{P}_{10} & \mathcal{P}_{11}
\end{array}\right)
$$


are projectors for all $t, s \in \mathbb{C}$.

Proof. The fact that $\mathcal{P}^{2}=\mathcal{P}$, coupled with the equations $\mathcal{P}_{00}^{2}=\mathcal{P}_{00}$ and $\mathcal{P}_{11}^{2}=\mathcal{P}_{11}$, imply that

$$
\begin{aligned}
& \mathcal{P}_{10} \mathcal{P}_{01}=0=\mathcal{P}_{01} \mathcal{P}_{10} \\
& \mathcal{P}_{00} \mathcal{P}_{10}+\mathcal{P}_{10} \mathcal{P}_{11}=\mathcal{P}_{10} \\
& \mathcal{P}_{01} \mathcal{P}_{00}+\mathcal{P}_{11} \mathcal{P}_{01}=\mathcal{P}_{01}
\end{aligned}
$$

From (32) we easily deduce that

$$
\mathcal{P}_{t, s}=\left(\begin{array}{cc}
\mathcal{P}_{00} & t \mathcal{P}_{10} \\
s \mathcal{P}_{01} & \mathcal{P}_{11}
\end{array}\right)
$$

is a projector, for $s, t \in \mathbb{C}$.

Remark 1. Evidently, $\mathcal{P}_{t, t}$ defines a homotopy through projectors, from $\mathcal{P}=$ $\mathcal{P}_{1,1}$ to a block diagonal matrix, $\mathcal{P}_{0,0}$.

In this section we consider the structure of the Calderon projector for a Dirac operator on a manifold with several boundary components. It is important to understand that any fundamental solution for $\partial$ leads to the construction of a Calderon projector. If $X$ is a $\operatorname{Spin}_{\mathbb{C}}$-manifold with boundary, we can assume that $X^{\circ}$ is a relatively compact open subset of larger

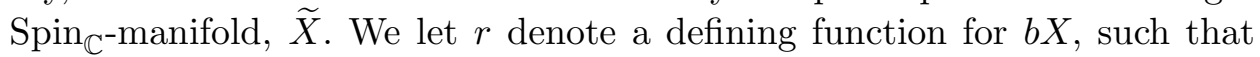
$d r \uparrow_{b X}$ is orthogonal to $T^{*} b X$ and $\|d r\|=1$. Let $\widetilde{\partial}$ denote the Dirac operator on $\widetilde{X}$. Assume that $Q$ is a fundamental solution defined on $\widetilde{X}$; thus if $s \in \mathcal{C}_{\mathrm{c}}^{\infty}(\widetilde{X} ; \not)$, then

$$
Q ð s=ð Q s=s .
$$

It is clear that $Q_{\mathcal{C}_{\mathrm{c}}^{\infty}}\left(\tilde{X} ; \$^{\prime}\right) \subset \mathcal{C}^{\infty}\left(\tilde{X} ; \phi^{\prime}\right)$ and therefore, by duality, we can extend $Q$ to act on $\mathcal{C}_{\mathrm{c}}^{-\infty}\left(\widetilde{X} ; \not^{\prime}\right)$.

Let $u \in \mathcal{C}^{\infty}(X ; \not)$, satisfy $\partial u=0$, and let $U$ denote the extension of $u$ by zero to all of $\widetilde{X}$. We see that $\precsim U=\boldsymbol{c}(d r) u \uparrow_{b X} \otimes \delta(r)$, and therefore:

$$
Q\left[\boldsymbol{c}(d r) u \uparrow_{b X} \otimes \delta(r)\right] \uparrow_{X}=u .
$$

More generally, if $f$ is a section of $\$ \uparrow_{b X}$, then

$$
F=Q[\boldsymbol{c}(d r) f \otimes \delta(r)]
$$

belongs to ker $\partial$ on $\widetilde{X} \backslash b X$. The analysis in Chapter 12 of [2] (for example) shows that $F$ has well defined limits as we approach $b X$, from either side, 
which we denote by $\mathcal{P}_{ \pm} f$. We use + to denote the limit from $X$ and - , the limit from $\widetilde{X} \backslash X$. The discussion above shows that $\mathcal{P}_{+}$acts as the identity on the boundary values of harmonic spinors defined in $X$.

Let $Q_{0}$ and $Q_{1}$ be two fundamental solutions defined in a neighborhood of $X$, and $\mathcal{P}_{0+}, \mathcal{P}_{1+}$ the Calderon projectors they define. Because two fundamental solutions differ by a smoothing operator, it follows that $(1-t) Q_{0}+t Q_{1}$ is also a fundamental solution for any $t \in[0,1]$. This implies that any pair of Calderon projectors are strongly isotopic:

Proposition 2. Let $\mathcal{P}_{0+}, \mathcal{P}_{1+}$ be Calderon projectors defined by fundamental solutions for $\partial_{X}$, then there is a smooth path, $\left\{\mathcal{P}_{t+}: t \in[0,1]\right\}$, in the space of pseudodifferential projections joining $\mathcal{P}_{0+}$ to $\mathcal{P}_{1+}$.

This result allows us to be a bit sloppy about which Calderon projector we are using.

In what follows we are usually more specific as to the origin of the fundamental solution. Indeed, $\widetilde{X}$ is usually taken to be a compact, closed manifold on which $\partial$ is invertible. The range of $\mathcal{P}_{-}$consists of the boundary values of harmonic spinors on $\widetilde{X} \backslash X$, and we have the jump formula:

$$
\mathcal{P}_{+}+\mathcal{P}_{-}=\mathrm{Id}
$$

Denote the Dirac operator on the invertible double, $\widehat{X}$, by $\widehat{\partial}$. Since $\widehat{\varnothing}$ is invertible, there is a fundamental solution, $\widehat{Q}$, defined on $\widehat{X}$, which is a classical pseudodifferential operator of order -1 . The Calderon projector, $\mathcal{P}_{+}$, for $\mathrm{\partial}$ on $X_{+} \simeq X$ is a pseudodifferential operator defined on $b X$ whose range consists of the boundary values of harmonic spinors on $X_{+}$, that is, solutions to

$$
ð \sigma=0
$$

in $\mathcal{C}^{\infty}\left(X_{+} ; \$^{\prime}\right)$. In [2] the fundamental solution $\widehat{Q}$ is used to construct a Calderon projector. As noted in (37) its nullspace consists of boundary values of harmonic spinors on $X_{-}$.

If $D \subset X_{+}$is any domain with smooth boundary, then the BooßBavnbeck-Wojciechowski construction applies, mutatis mutandis, to construct a Calderon projector, $\mathcal{P}_{D_{+}}$, defined on $b D$. The range of $\mathcal{P}_{D_{+}}$consists of the boundary values of harmonic spinors defined on $D$, and its nullspace consists of boundary values of harmonic spinors defined on the complement $\widehat{X} \backslash D$. If we denote this complement by $D_{-}$, then this statement is simply the identity:

$$
\mathcal{P}_{D_{+}}+\mathcal{P}_{D_{-}}=\mathrm{Id}
$$

The proof of the following result is now quite simple: 


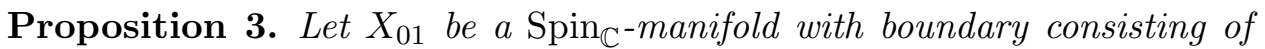
two components, $Y_{0}, Y_{1}$. Suppose that $\bar{Y}_{0}$ is the boundary of a $\operatorname{Spin}_{\mathbb{C}}$-manifold with boundary $X_{0}$. There is a Calderon projector, $\mathcal{P}$, for $\widetilde{\partial}_{X_{01}}$, so that with respect to the splitting in (30), the diagonal terms satisfy:

$$
\mathcal{P}_{00}^{2}=\mathcal{P}_{00} \text { and } \mathcal{P}_{11}^{2}=\mathcal{P}_{11}
$$

Proof. We let $X_{1}$ denote the $\operatorname{Spin}_{\mathbb{C}}$-manifold obtained by gluing $X_{0}$ to $X_{01}$. To do this gluing it may be necessary to glue collars onto $X_{0}$ and $X_{01}$, in

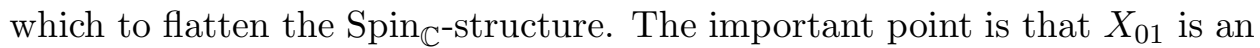
open subset of $X_{1}$. We can then double $X_{1}$ across its remaining boundary, to obtain the invertible double $\widehat{X}_{1}$. Let $\widehat{Q}$ denote the fundamental solution for $\widehat{\partial}$ on $\widehat{X}_{1}$. Using this fundamental solution we construct Calderon projectors for $X_{01}, X_{1}$ and $\left(X_{1} \backslash X_{01}\right)^{\circ}$, which we denote by $\mathcal{P}, \mathcal{P}_{1}$, and $\mathcal{P}_{0}$. In light of the construction of a Calderon projector as a limit, and (39), a moments thought shows that the block decomposition of $\mathcal{P}$ takes the form:

$$
\mathcal{P}=\left(\begin{array}{cc}
\mathrm{Id}-\mathcal{P}_{0} & P_{10} \\
P_{01} & \mathcal{P}_{1}
\end{array}\right)
$$

As $\mathcal{P}_{0}$ and $\mathcal{P}_{1}$ are projectors, the assertion of the proposition follows.

Propositions 1 and 3 imply:

Corollary 1. Under the hypotheses of Proposition 3 , the Calderon projector can be deformed through projectors to

$$
\mathcal{P}_{d}=\left(\begin{array}{cc}
\mathrm{Id}-\mathcal{P}_{0} & 0 \\
0 & \mathcal{P}_{1}
\end{array}\right)
$$

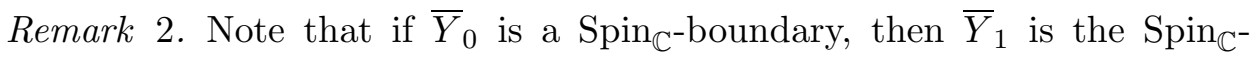
boundary of $\bar{X}_{1}$.

These results have a natural generalization when $X_{0}$ has many boundary components. Suppose that $b X_{0}$ has several components, $Y_{1}, \ldots, Y_{N}$. We group these boundary components into disjoint (non-empty) subsets

$$
\begin{aligned}
& Y^{j}=\amalg_{l=m_{j}}^{m_{j+1}-1} Y_{l}, \quad j=1, \ldots, J, \text { where } \\
& 1=m_{1}<m_{2}<\cdots<m_{J}<m_{J+1}=N+1,
\end{aligned}
$$

with the property that each collection $\bar{Y}^{j}$ is the boundary of a $\operatorname{Spin}_{\mathbb{C}^{-}}$ manifold, $X_{j}$. Gluing along these collections of boundary components we 


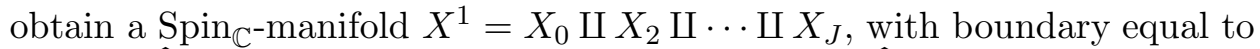
$Y^{1}$. Let $\widehat{X}^{1}$ denote the invertible double of $X^{1}$, and $\widehat{Q}$ its fundamental solution. There is a Calderon projector for $X_{0}$ that can be deformed to a block diagonal matrix, with one block for each collection of boundary components $Y^{j}$.

For each $2 \leq j \leq J$, let $\mathcal{P}^{j}$ denote the Calderon projector, defined by $\widehat{Q}$, for the manifold $X_{j}$, and $\mathcal{P}^{1}$ the Calderon projector for $X^{1}$. For each $2 \leq k \leq J$, let $\widetilde{\mathcal{P}}^{k}$ denote the Calderon projector, defined by $\widehat{Q}$, for the manifold $\widetilde{X}_{k}=X_{0} \amalg X_{k} \amalg \cdots \amalg X_{J}$. With these preliminaries we can state the following theorem.

Theorem 2. With $X_{0}, Y^{1}, \ldots, Y^{J}$ as above, the Calderon projector, $\mathcal{P}^{0}$, for $X_{0}$, can be deformed through projectors to the block diagonal matrix:

$$
\mathcal{P}_{0}^{0}=\left(\begin{array}{ccccc}
\mathcal{P}^{1} & 0 & & \cdots & 0 \\
0 & \operatorname{Id}-\mathcal{P}^{2} & 0 & \cdots & \vdots \\
\vdots & & \ddots & & 0 \\
0 & \cdots & & & \mathrm{Id}-\mathcal{P}^{J}
\end{array}\right)
$$

Proof. We split $\$ \uparrow_{b X}$ into

$$
\$ \uparrow_{b X}=\$ \uparrow_{Y^{1} \amalg \cdots \amalg Y^{J-1}} \oplus \$ \uparrow_{Y^{J}}
$$

In the notation introduced before the theorem the projector, $\mathcal{P}^{0}$ then takes the form

$$
\mathcal{P}^{0}=\left(\begin{array}{cc}
\widetilde{\mathcal{P}}^{J} & A_{J} \\
B_{J} & \mathrm{Id}-\mathcal{P}^{J}
\end{array}\right)
$$

Here $A_{J}, B_{J}$ are smoothing operators. As $\mathcal{P}^{0}, \widetilde{\mathcal{P}}^{J}$ and $\mathcal{P}^{J}$ are all projectors, Proposition 1 shows that

$$
\mathcal{P}_{1 t}^{0}=\mathcal{P}^{0}=\left(\begin{array}{cc}
\widetilde{\mathcal{P}}^{J} & t A_{J} \\
t B_{J} & \mathrm{Id}-\mathcal{P}^{J}
\end{array}\right)
$$

is a one parameter family of projectors, and we can therefore deform to

$$
\mathcal{P}_{10}^{0}=\left(\begin{array}{cc}
\widetilde{\mathcal{P}}^{J} & 0 \\
0 & \mathrm{Id}-\mathcal{P}^{J}
\end{array}\right) .
$$

For $3 \leq k \leq J$, we see that

$$
\$ \uparrow_{b \widetilde{X}^{k}} \simeq \$ \uparrow_{Y^{1} \amalg \cdots \amalg Y^{k-2}} \oplus \$ \uparrow_{Y^{k-1}}
$$


and, with respect to this splitting, the projector $\widetilde{\mathcal{P}}^{k}$, is of the form

$$
\widetilde{\mathcal{P}}^{k}=\left(\begin{array}{cc}
\widetilde{\mathcal{P}}^{k-1} & A_{k-1} \\
B_{k-1} & \mathrm{Id}-\mathcal{P}^{k-1}
\end{array}\right)
$$

Repeating this argument recursively, along with the fact that $\mathcal{P}^{1}=\widetilde{\mathcal{P}}^{2}$, leads to a homotopy through projectors (with constant block diagonal) from $\mathcal{P}^{0}$ to $\mathcal{P}_{0}^{0}$.

\section{Analysis on manifold with several boundary com- ponents}

In this section we study the index of the $\operatorname{Spin}_{\mathbb{C}^{-D i r a c}}$ operator on a manifold with several boundary components, some pseudoconvex and some pseudoconcave. For example, let $X_{01}$, have two boundary components, $Y_{0}, Y_{1}$. We

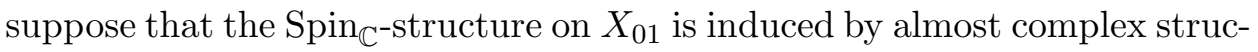
tures in neighborhoods of its boundary components. We also assume that $Y_{1}$ is pseudoconvex and $Y_{0}$ is pseudoconcave, with respect to the corresponding almost complex structures. The boundary components, $Y_{0}, Y_{1}$ are contact manifolds. We let $\mathcal{S}_{0}, \mathcal{S}_{1}$ be generalized Szegö projectors defined on $\left(Y_{0}, H_{0}\right)$, $\left(Y_{1}, H_{1}\right)$, respectively. Along with the almost complex structures, these define projectors, $\mathcal{R}_{0+}, \mathcal{R}_{1+}$ acting on sections of the spin-bundle restricted to the boundary. Let $\partial_{X_{01}}$ denote the $\operatorname{Spin}_{\mathbb{C}^{-D}}$ Dirac operator on $X_{01}$. We let $\left(\partial_{X_{01}},\left[\left(\mathrm{Id}-\mathcal{R}_{0+}\right), \mathcal{R}_{1+}\right]\right)$ denote the $\operatorname{Spin}_{\mathbb{C}}$-Dirac operator acting on smooth spinors $\sigma$, which satisfy:

$$
\left(\mathrm{Id}-\mathcal{R}_{0+}\right)\left[\sigma \uparrow_{Y_{0}}\right]=0 \text { and } \mathcal{R}_{1+}\left[\sigma \uparrow_{Y_{1}}\right]=0 .
$$

In [8, 9] we established the analytic properties of these boundary value problems by studying the comparison operator:

$$
\mathcal{T}=\mathcal{R} \mathcal{P}+(\mathrm{Id}-\mathcal{R})(\operatorname{Id}-\mathcal{P})
$$

here $\mathcal{R}$ is either the pseudoconvex or pseudoconcave modification of the $\bar{\partial}$ Neumann condition and $\mathcal{P}$ is a Calderon projector for $\precsim$. The analytic results follow from the existence of a parametrix, $\mathcal{U}$, for $\mathcal{T}$ satisfying

$$
\begin{aligned}
\mathcal{T U} & =\mathrm{Id}-K_{1} \\
\mathcal{U T} & =\mathrm{Id}-K_{2},
\end{aligned}
$$

where $K_{1}, K_{2}$ are smoothing operators on $b X$. The operator $\mathcal{U}$ belongs to the extended Heisenberg calculus on $b X$ and its construction is entirely 
microlocal. The input from the $\operatorname{Spin}_{\mathbb{C}^{-} \text {structure/Spin }} \mathbb{C}^{-D i r a c}$ operator is that coming from the principal symbol of the Calderon projector.

Thus far, we have only given the complete details of this construction for $\left(ð, \mathcal{R}_{+}\right)$on a strictly pseudoconvex $\operatorname{Spin}_{\mathbb{C}^{-}}$manifold. Because these results rest entirely upon the construction of $\mathcal{U}$, they also hold for Id $-\mathcal{R}_{+}$ on a strictly pseudoconcave manifold: Clearly $\mathcal{T}$ is symmetric in $\mathcal{R}$ and Id $-\mathcal{R}$. When combined with the fact that the construction of $\mathcal{U}$ uses only the principal symbol of the Calderon projector, and $\sigma_{0}\left(\mathcal{P}_{-}\right)=\sigma_{0}\left(\operatorname{Id}-\mathcal{P}_{+}\right)$, we see that it makes no difference whether we are working on the boundary of a pseudoconvex manifold using the boundary condition $\mathcal{R}_{+}$, or on a pseudoconcave manifold using the boundary condition Id $-\mathcal{R}_{+}$.

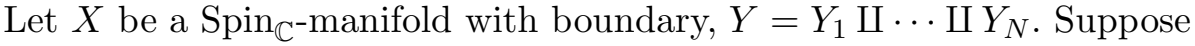
that an almost complex structure is defined in a neighborhood of the each boundary component, inducing the given $\operatorname{Spin}_{\mathbb{C}^{-} \text {-structure, such that each }}$ boundary component is either strictly pseudoconvex on strictly pseudoconcave. Let $\mathcal{P}_{+}$be the Calderon projector defined on $X$ by including $X$ into a compact $\operatorname{Spin}_{\mathbb{C}}$-manifold, $\widetilde{X}$ with an invertible Dirac operator. Let $\mathcal{P}_{-}$ denote the Calderon projector for $\widetilde{X} \backslash X$; it is important that

$$
\mathcal{P}_{+}+\mathcal{P}_{-}=\mathrm{Id}
$$

For a Calderon projector defined by embedding $X$ into a compact manifold with invertible Dirac-operator the following result, which is Proposition 11 in [9], holds:

Proposition 4. Let $X$ be a $\operatorname{Spin}_{\mathbb{C}}$-manifold with boundary embedded into $\widetilde{X}$ a compact $\operatorname{Spin}_{\mathbb{C}}$-manifold with invertible Dirac operator. Let $t$ be a defining function for $b X$ such that $t<0$ on $X, \operatorname{grad}_{g} t$ is orthogonal to $T b X$ and $\|d t\|=1$ along $b X$. If $\mathcal{P}_{ \pm}^{\mathrm{eo}}$ are Calderon projectors defined by the fundamental solution to ठे on $\widetilde{X}$ then

$$
\mathcal{P}_{ \pm}^{\mathrm{eo}}=\boldsymbol{c}( \pm d t) \mathcal{P}_{\mp}^{\mathrm{oe}} \boldsymbol{c}( \pm d t)^{-1}
$$

Proof. In the statement of Proposition 11 in [9] it is assumed that $\tilde{X}$ is an invertible double for $X$, however this hypothesis is not used in the proof. All that is needed is the assumption that the Dirac operator on $\widetilde{X}$ is invertible and the Calderon projector is constructed using the fundamental solution defined on $\widetilde{X}$.

With respect to the splitting

$$
\not \$ \uparrow_{b X}=\$ \uparrow_{Y_{1}} \oplus \cdots \oplus \$_{Y_{N}}
$$


the Calderon projector takes the form:

$$
\mathcal{P}=\left(\begin{array}{cccc}
\mathcal{P}_{11} & \mathcal{P}_{12} & \cdots & \mathcal{P}_{1 n} \\
\mathcal{P}_{21} & \mathcal{P}_{22} & \cdots & \mathcal{P}_{2 n} \\
\vdots & \vdots & & \vdots \\
\mathcal{P}_{n 1} & \mathcal{P}_{n 2} & \cdots & \mathcal{P}_{n n}
\end{array}\right)
$$

Usually we will make assumptions that imply $\mathcal{P}_{j j}^{2}=\mathcal{P}_{j j}$ for $1 \leq j \leq N$, but in all cases $\mathcal{P}_{j j}^{2}-\mathcal{P}_{j j}$ and $\mathcal{P}_{j k}$ for $j \neq k$ are smoothing operators. For each $j$ we choose a generalized Szegö projector, $\mathcal{S}_{j} \in \Psi_{H_{j}}^{0}\left(Y_{j}\right)$. Let $\mathcal{R}_{j+}$ denote the modified pseudoconvex $\bar{\partial}$-Neumann condition defined by $\mathcal{S}_{j}$. For a pseudoconvex boundary component, $Y_{j}$, we let

$$
\mathcal{T}_{j}^{+}=\mathcal{R}_{j+} \mathcal{P}_{j j}+\left(\mathrm{Id}-\mathcal{R}_{j+}\right)\left(\mathrm{Id}-\mathcal{P}_{j j}\right),
$$

for a pseudoconcave boundary component, $Y_{k}$, we let

$$
\mathcal{T}_{k}^{-}=\left(\mathrm{Id}-\mathcal{R}_{k+}\right) \mathcal{P}_{k k}+\mathcal{R}_{k+}\left(\mathrm{Id}-\mathcal{P}_{k k}\right) .
$$

Define the function $\epsilon_{j}=+$ if $Y_{j}$ is pseudoconvex and - otherwise.

The remarks above easily imply the following result.

Proposition 5. For each boundary component, $Y_{j}$ the operator $\mathcal{T}_{j}^{\epsilon_{j}}$ is an elliptic element in the extended Heisenberg algebra. There is a parametrix $\mathcal{U}_{j}^{\epsilon_{j}}$ so that, for smoothing operators $K_{j 1}, K_{j 2}$, we have

$$
\mathcal{T}_{j}^{\epsilon_{j}} \mathcal{U}_{j}^{\epsilon_{j}}=\mathrm{Id}-K_{j 1} \text { and } \mathcal{U}_{j}^{\epsilon_{j}} \mathcal{T}_{j}^{\epsilon_{j}}=\mathrm{Id}-K_{j 2} .
$$

Now we order the boundary components so that $Y_{1}, \ldots, Y_{L}$ are strictly pseudoconcave and $Y_{L+1}, \ldots, Y_{N}$ are strictly pseudoconvex. Set

$$
\mathcal{R}=\left(\begin{array}{ccccccc}
\mathrm{Id}-\mathcal{R}_{1+} & 0 & \cdots & 0 & \cdots & & 0 \\
\vdots & \ddots & & \vdots & & & \vdots \\
0 & \cdots & \mathrm{Id}-\mathcal{R}_{L+} & 0 & \cdots & & 0 \\
0 & \cdots & 0 & \mathcal{R}_{(L+1)+} & 0 & \cdots & \vdots \\
\vdots & & & & \ddots & & 0 \\
0 & \cdots & 0 & 0 & \cdots & 0 & \mathcal{R}_{N+}
\end{array}\right),
$$

and let

$$
\mathcal{T}=\mathcal{R} \mathcal{P}+(\mathrm{Id}-\mathcal{R})(\operatorname{Id}-\mathcal{P})
$$

The following relationship between the chiral parts $\mathcal{R}^{\mathrm{e}}$ and $\mathcal{R}^{\mathrm{o}}$ is a consequence of the formal self adjointness of $\mathcal{R}$; it is proved in [9]. 
Proposition 6. The chiral parts satisfy:

$$
\mathcal{R}^{\mathrm{e}}=\boldsymbol{c}(d t)\left(\mathrm{Id}-\mathcal{R}^{\mathrm{o}}\right) \boldsymbol{c}(d t)^{-1}
$$

If we define $\mathcal{U}$ to be the diagonal matrix with diagonal

$$
\mathcal{U}=\operatorname{diag}\left(\mathcal{U}_{1}^{-}, \ldots, \mathcal{U}_{L}^{-}, \mathcal{U}_{L+1}^{+}, \ldots, \mathcal{U}_{N}^{+}\right),
$$

then Proposition 5, and the fact that the off-diagonal elements in $\mathcal{P}$ are smoothing operators implies the following basic result:

Theorem 3. The operator $\mathcal{U}$ is a parametrix for $\mathcal{T}$.

Proof. Let $\mathcal{P}_{d}$ denote the diagonal of $\mathcal{P}$, and $\mathcal{P}_{\text {od }}=\mathcal{P}-\mathcal{P}_{d}$. If we let $\mathcal{T}_{d}=$ $\mathcal{R} \mathcal{P}_{d}+(\mathrm{Id}-\mathcal{R})\left(\mathrm{Id}-\mathcal{P}_{d}\right)$, then Proposition 5 implies that

$$
\mathcal{U} \mathcal{T}_{d}-\operatorname{Id} \text { and } \mathcal{T}_{d} \mathcal{U}-\mathrm{Id}
$$

are smoothing operators. As $\mathcal{T}-\mathcal{T}_{d}=(2 \mathcal{R}-\mathrm{Id}) \mathcal{P}_{\text {od }}$ is a smoothing operator it follows immediately that $K_{1}$ and $K_{2}$ in

$$
\mathcal{T U}=\operatorname{Id}-K_{1} \text { and } \mathcal{U} \mathcal{T}=\operatorname{Id}-K_{2}
$$

are also smoothing operators.

In the case that the diagonal of $\mathcal{P}$ is a projector, this argument gives a stronger result.

Corollary 2. Suppose that $\mathcal{P}_{d}^{2}=\mathcal{P}_{d}$; define

$$
\mathcal{P}_{t}=\mathcal{P}-t \mathcal{P}_{\text {od }} \text { and } \mathcal{T}_{t}=\mathcal{R} \mathcal{P}_{t}+(\operatorname{Id}-\mathcal{R})\left(\operatorname{Id}-\mathcal{P}_{t}\right)
$$

For each $t, \mathcal{P}_{t}$ is a projector, and $\mathcal{U}$ is a parametrix for $\mathcal{T}_{t}$, with

$$
\mathcal{T}_{t} \mathcal{U}=\mathrm{Id}-K_{1 t} \text { and } \mathcal{U} \mathcal{T}_{t}=\mathrm{Id}-K_{2 t} .
$$

The operators $\left\{\left(K_{1 t}, K_{2 t}\right): t \in[0,1]\right\}$ are a smooth family of smoothing operators.

Remark 3. Note that

$$
\mathcal{P}_{0}=\mathcal{P} \text { and } \mathcal{P}_{1}=\mathcal{P}_{d}
$$




\section{The relative index formula}

Recall that if $\sigma$ and its distributional derivative, $\precsim \sigma$, both belong to $L^{2}(X ; \not)$, then $\sigma$ has a well defined restriction to $b X$ as an element of the Sobolev space $H^{-\frac{1}{2}}\left(b X ; \$ \uparrow_{b X}\right)$. Theorem 3 combined with the arguments in [9] prove the following result:

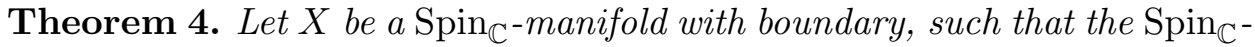
structure is defined in a neighborhood of $b X$ by an almost complex structure, making each boundary component of $X$ either strictly pseudoconvex or strictly pseudoconcave. If we define the domain for $\mathrm{\partial}$ to be

$$
\left\{\sigma \in L^{2}(X ; \not): \succsim \sigma \in L^{2}(X ; \not), \mathcal{R}\left[\sigma \uparrow_{b X}\right]=0\right\},
$$

where $\mathcal{R}$ is defined as in (61), then $\partial$ is a Fredholm operator. There is a constant $C$ so that if $\sigma$ satisfies these conditions, then

$$
\|\sigma\|_{H^{\frac{1}{2}(X)}} \leq C\left[\|ð \sigma\|_{L^{2}(X)}+\|\sigma\|_{L^{2}(X)}\right] .
$$

The chiral restrictions $\mathrm{J}^{\mathrm{eo}}$ are Fredholm and their $L^{2}$-adjoints satisfy

$$
\left[\left(\check{\partial}^{\mathrm{eo}}, \mathcal{R}^{\mathrm{eo}}\right)\right]^{*}=\overline{\left(\check{(\beth}^{\mathrm{oe}}, \mathcal{R}^{\mathrm{oe}}\right)} \text {. }
$$

Remark 4. Indeed, there are also higher norm estimates: For each $s \geq 0$, there is a constant $C_{s}$ so that if $\sigma \in L^{2}, \precsim \sigma \in H^{s}$, and $\mathcal{R}\left[\sigma \uparrow_{b X}\right]=0$, then $\sigma \in H^{s+\frac{1}{2}}$, and

$$
\|\sigma\|_{H^{s+\frac{1}{2}}} \leq C_{s}\left[\|\mathrm{\partial} \sigma\|_{H^{s}}+\|\sigma\|_{L^{2}}\right] .
$$

These estimates imply that the null-space of $\partial$ is contained in $\mathcal{C}^{\infty}\left(X ; \$^{\prime}\right)$.

As in our earlier papers, the indices of $\left(\mathrm{J}^{\mathrm{eo}}, \mathcal{R}^{\mathrm{eo}}\right)$ can be computed as the relative indices on the boundary between $\mathcal{P}^{\text {eo }}$ and $\mathcal{R}^{\text {eo }}$. Theorem 3 shows that $\mathcal{P}^{\mathrm{eo}}$ and $\mathcal{R}^{\mathrm{eo}}$ are a tame Fredholm pair, and therefore the relative index can be computed as the index of:

$$
\mathrm{R}-\operatorname{Ind}\left(\mathcal{P}^{\mathrm{eo}}, \mathcal{R}^{\mathrm{eo}}\right)=\operatorname{Ind}\left[\mathcal{R}^{\mathrm{eo}}: \mathcal{P}^{\mathrm{eo}} \mathcal{C}^{\infty}\left(b X ; \not \uparrow_{b X}\right) \longrightarrow \mathcal{R}^{\mathrm{eo}} \mathcal{C}^{\infty}\left(b X ; \not \uparrow_{b X}\right)\right] .
$$

Theorem 5. Let $X$ be a compact $\operatorname{Spin}_{\mathbb{C}}$-manifold as in Theorem 4. Suppose that $\mathcal{P}$ is a Calderon projector for $\partial_{X}$, which satisfies

$$
\mathcal{P}^{\mathrm{e} *}=\boldsymbol{c}(d t)\left(\mathrm{Id}-\mathcal{P}^{\mathrm{o}}\right) \boldsymbol{c}(d t)^{-1} .
$$

If $\mathcal{R}$ is a projector acting on sections of $\not \$\lceil X X$ as in (61), then

$$
\operatorname{Ind}\left(\check{\partial}^{\mathrm{eo}}, \mathcal{R}^{\mathrm{eo}}\right)=\mathrm{R}-\operatorname{Ind}\left(\mathcal{P}^{\mathrm{eo}}, \mathcal{R}^{\mathrm{eo}}\right) \text {. }
$$


Remark 5. If the Calderon projector is defined by embedding $X$ into $\widetilde{X}$, a closed compact $\operatorname{Spin}_{\mathbb{C}}$-manifold, with invertible Dirac operator, then the relation (75) follows from Proposition 4 and (54).

Proof. We give the proof for the even case, the odd case is essentially identical. The null-space of $\left(\check{\beth}^{\mathrm{e}}, \mathcal{R}^{\mathrm{e}}\right)$ consists of smooth sections $\sigma$ of $\not \$^{\mathrm{e}}$ satisfying:

$$
\check{\partial}^{\mathrm{e}} \sigma=0 \text { and } \mathcal{R}^{\mathrm{e}}\left[\sigma \uparrow_{b X}\right]=0 .
$$

It is clear that $\mathcal{P}^{\mathrm{e}}\left[\sigma \uparrow_{b X}\right]=\sigma \uparrow_{b X}$, and therefore $\sigma \uparrow_{b X}$ belongs to the nullspace of $\mathcal{R}^{\mathrm{e}}$ acting on the range of $\mathcal{P}^{\mathrm{e}}$. On the other hand, if $s \in$ range $\mathcal{P}^{\mathrm{e}}$ and $\mathcal{R}^{\mathrm{e}} s=0$, then there is a unique harmonic spinor $\sigma$, with $\sigma \uparrow_{b X}=s$. This shows that the null-space of $\left(\check{\beth}^{\mathrm{e}}, \mathcal{R}^{\mathrm{e}}\right)$ is isomorphic to the null-space of the restriction in (74).

The co-kernel of $\mathcal{R}^{\mathrm{e}} \mathcal{P}^{\mathrm{e}}$ is isomorphic to the null-space of

$$
\mathcal{P}^{\mathrm{e} *} \text { : range } \mathcal{R}^{\mathrm{e}} \longrightarrow \text { range } \mathcal{P}^{\mathrm{e} *} \text {. }
$$

Equation (63) implies that the range of $\mathcal{R}^{\mathrm{e}}$ is $\boldsymbol{c}(d t)$ applied to the null-space of $\mathcal{R}^{\mathrm{o}}$; this, along with (175), shows that the co-kernel of $\mathcal{R}^{\mathrm{e}} \mathcal{P}^{\mathrm{e}}$ is isomorphic to the intersection of the null-space of $\mathcal{R}^{\circ}$ with the range of $\mathcal{P}^{\circ}$. By the first part of the argument, this intersection is isomorphic to $\operatorname{ker}\left(\widetilde{\partial}^{\circ}, \mathcal{R}^{\circ}\right)$. Applying the last statement of Theorem 4, we complete the proof of the theorem.

Using general properties of tame Fredholm pairs it follows that the relative index can be computed as a difference of traces.

Corollary 3. Suppose that the parametrix $\mathcal{U}$ for $\mathcal{T}$ satisfies (66), then

$$
\operatorname{Ind}\left(\check{\partial}^{\mathrm{eo}}, \mathcal{R}^{\mathrm{eo}}\right)=\mathrm{R}-\operatorname{Ind}\left(\mathcal{P}^{\mathrm{eo}}, \mathcal{R}^{\mathrm{eo}}\right)=\operatorname{tr}\left(\mathcal{P}^{\mathrm{eo}} K_{2}^{\mathrm{eo}} \mathcal{P}^{\mathrm{eo}}\right)-\operatorname{tr}\left(\mathcal{R}^{\mathrm{eo}} K_{1}^{\mathrm{eo}} \mathcal{R}^{\mathrm{eo}}\right)
$$

Proof. Because $\mathcal{P}^{\mathrm{eo}}$ and $\mathcal{R}^{\mathrm{eo}}$ are tame Fredholm pairs, this is an immediate consequence of Theorem 15 in [9].

As in [9] the relative index formula has a useful corollary:

Corollary 4. Let $X$ be a compact $\operatorname{Spin}_{\mathbb{C}}$-manifold with boundary as in Theorem 4 and $\mathcal{R}$ a modified $\bar{\partial}-N e u m a n n$ boundary condition as in (61). If $\mathcal{P}$ is a Calderon projector for $\partial_{X}$, then

$$
\operatorname{R}-\operatorname{Ind}\left(\mathcal{P}^{\mathrm{eo}}, \mathcal{R}^{\mathrm{eo}}\right)=-\mathrm{R}-\operatorname{Ind}\left(\left(\mathrm{Id}-\mathcal{P}^{\mathrm{eo}}\right),\left(\mathrm{Id}-\mathcal{R}^{\mathrm{eo}}\right)\right) .
$$


Proof. It follows from Proposition 3 and Corollary 3 that the relative indices in (80) do not depend on the choice of Calderon projector, and therefore we can assume that $\mathcal{P}$ is defined using the invertible double construction. As it relies only on very general properties of the Calderon projector, and the invertible double construction, the argument used to prove Corollary 5 in 9 ] applies, with minor changes, to establish (80).

In the case that the diagonal of $\mathcal{P}, \mathcal{P}_{d}$, is itself a projector, Corollary 2 shows that, for each $t \in[0,1],\left(\mathcal{P}_{t}, \mathcal{R}^{\mathrm{eo}}\right)$, where $\mathcal{P}_{t}^{\mathrm{eo}}=\mathcal{P}^{\mathrm{eo}}-t \mathcal{P}_{\text {od }}^{\mathrm{eo}}$, is a tame Fredholm pair. The index of these pairs can also be computed by evaluating a trace:

$$
\mathrm{R}-\operatorname{Ind}\left(\mathcal{P}_{t}^{\mathrm{eo}}, \mathcal{R}^{\mathrm{eo}}\right)=\operatorname{tr}\left(\mathcal{P}_{t}^{\mathrm{eo}} K_{2 t}^{\mathrm{eo}} \mathcal{P}_{t}^{\mathrm{eo}}\right)-\operatorname{tr}\left(\mathcal{R}^{\mathrm{eo}} K_{1 t}^{\mathrm{eo}} \mathcal{R}^{\mathrm{eo}}\right)
$$

The operators on the right hand side of (81) are smoothing operators, depending smoothly on $t$, hence the traces depend smoothly on $t$ as well. As the difference is an integer it must be constant. This proves the following result.

Theorem 6. If $X, \mathcal{P}, \mathcal{R}$ satisfy the hypotheses of Theorem 5 , and the diagonal of the Calderon projector is itself a projector, then

$$
\operatorname{Ind}\left(\check{\partial}^{\mathrm{eo}}, \mathcal{R}^{\mathrm{eo}}\right)=\mathrm{R}-\operatorname{Ind}\left(\mathcal{P}_{d}^{\mathrm{eo}}, \mathcal{R}^{\mathrm{eo}}\right) .
$$

This result is our basic tool for studying the gluing properties of the indices of sub-elliptic boundary value problems for $\precsim$.

\section{Gluing formulæ for the index of $\varnothing$}

We now consider the behavior of the index of $\partial$ with modified $\bar{\partial}$-Neumann conditions under gluing operations. This approach was implicitly used in [7], though we did not directly address the analytic properties of boundary value problems on manifolds with several ends. Under this rubric there is a huge multiplicity of possible situations that one might consider, in this section

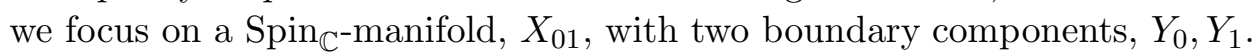

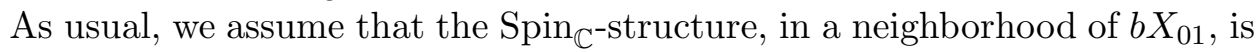
induced by an almost structure, and that $Y_{0}, Y_{1}$ are contact manifolds, with $Y_{0}$ strictly pseudoconcave and $Y_{1}$ strictly pseudoconvex.

Let $\mathcal{S}_{0}, \mathcal{S}_{1}$ be generalized Szegö projectors defined on $Y_{0}, Y_{1}$ respectively and $\mathcal{R}_{0}, \mathcal{R}_{1}$, the pseudoconvex, modified $\bar{\partial}$-Neumann boundary conditions 
they define. As it is the case of principal interest in applications to complex analysis, we often assume that $\bar{Y}_{0}$ is also the pseudoconvex bound-

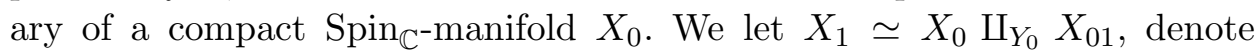

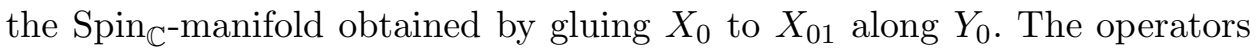
$\left(\partial_{X_{0}}, \mathcal{R}_{0}\right),\left(\partial_{X_{1}}, \mathcal{R}_{1}\right)$ are Fredholm, as is $\left(\partial_{X_{01}},\left[\mathrm{Id}-\mathcal{R}_{0}, \mathcal{R}_{1}\right]\right)$. Our basic result is a gluing formula for $\operatorname{Ind}\left(\check{\partial}_{X_{1}}, \mathcal{R}_{1}\right)$.

Theorem 7. Let $X_{0}, X_{01}$ and $X_{1}$ be as above, with $\mathcal{R}_{0}, \mathcal{R}_{1}$ modified pseudoconvex $\bar{\partial}$-Neumann conditions. The indices satisfy the following relation:

$$
\operatorname{Ind}\left(\varlimsup_{X_{1}}^{\mathrm{eo}}, \mathcal{R}_{1}^{\mathrm{eo}}\right)=\operatorname{Ind}\left(\varlimsup_{X_{0}}^{\mathrm{eo}}, \mathcal{R}_{0}\right)+\operatorname{Ind}\left(\varlimsup_{X_{01}}^{\mathrm{eo}},\left[\left(\operatorname{Id}-\mathcal{R}_{0}^{\mathrm{eo}}\right), \mathcal{R}_{1}^{\mathrm{eo}}\right]\right) .
$$

Proof. To prove this formula, we express the various indices in terms of relative indices on the boundaries. Let $\widehat{X}_{1}$ denote the invertible double of $X_{1}$, and $\widehat{Q}_{1}$ the fundamental solution for $\widetilde{\partial}_{\widehat{X}_{1}}$. Let $\mathcal{P}_{0}, \mathcal{P}_{1}$ be Calderon projectors, for $\partial_{X_{0}}, \partial_{X_{1}}$, respectively, defined by $\widehat{Q}_{1}$. Finally let $\mathcal{P}_{01}$ be the Calderon projector for $\widetilde{\partial}_{X_{01}}$ defined by $\widehat{Q}_{1}$. The discussion in Section 2 shows that

$$
\mathcal{P}_{01}=\left(\begin{array}{cc}
\mathrm{Id}-\mathcal{P}_{0} & P_{10} \\
P_{01} & \mathcal{P}_{1}
\end{array}\right)
$$

and therefore the diagonal of $\mathcal{P}_{01}$ is itself a projector. Theorem 5 shows that

$$
\begin{aligned}
& \operatorname{Ind}\left(\left(_{X_{0}}^{\mathrm{eo}}, \mathcal{R}_{0}\right)+\operatorname{Ind}\left(\check{\partial}_{X_{01}}^{\mathrm{eo}},\left[\left(\operatorname{Id}-\mathcal{R}_{0}^{\mathrm{eo}}\right), \mathcal{R}_{1}^{\mathrm{eo}}\right]\right)=\right. \\
& \quad \operatorname{R}-\operatorname{Ind}\left(\mathcal{P}^{\mathrm{eo}}, \mathcal{R}_{0}\right)+\operatorname{R}-\operatorname{Ind}\left(\mathcal{P}_{01}^{\mathrm{eo}},\left[\left(\operatorname{Id}-\mathcal{R}_{0}^{\mathrm{eo}}\right), \mathcal{R}_{1}^{\mathrm{eo}}\right]\right) .
\end{aligned}
$$

Theorem [6] applies to show that the second term on the right hand side of (85) can be replaced by

$$
\begin{aligned}
\operatorname{R}-\operatorname{Ind}\left(\mathcal{P}_{01}^{\mathrm{eo}},\left[\left(\operatorname{Id}-\mathcal{R}_{0}^{\mathrm{eo}}\right), \mathcal{R}_{1}^{\mathrm{eo}}\right]\right) & =\operatorname{R}-\operatorname{Ind}\left(\left[\left(\operatorname{Id}-\mathcal{P}_{0}^{\mathrm{eo}}\right), \mathcal{P}_{1}^{\mathrm{eo}}\right],\left[\left(\operatorname{Id}-\mathcal{R}_{0}^{\mathrm{eo}}\right), \mathcal{R}_{1}^{\mathrm{eo}}\right]\right) \\
& =\operatorname{R}-\operatorname{Ind}\left(\left(\operatorname{Id}-\mathcal{P}_{0}^{\mathrm{eo}}\right),\left(\operatorname{Id}-\mathcal{R}_{0}^{\mathrm{eo}}\right)\right)+\operatorname{R}-\operatorname{Ind}\left(\mathcal{P}_{1}^{\mathrm{eo}}, \mathcal{R}_{1}^{\mathrm{eo}}\right) .
\end{aligned}
$$

Finally we apply Corollary 5 from [9] to replace $\mathrm{R}-\operatorname{Ind}\left(\left(\operatorname{Id}-\mathcal{P}_{0}^{\text {eo }}\right),\left(\operatorname{Id}-\mathcal{R}_{0}^{\text {eo }}\right)\right)$ with $-\mathrm{R}-\operatorname{Ind}\left(\mathcal{P}_{0}^{\mathrm{eo}}, \mathcal{R}_{0}^{\mathrm{eo}}\right)$. Once again applying Theorem 5 we obtain

$$
\operatorname{Ind}\left(\check{\varpi}_{X_{0}}^{\mathrm{eo}}, \mathcal{R}_{0}\right)+\operatorname{Ind}\left(\check{\varpi}_{X_{01}}^{\mathrm{eo}},\left[\left(\mathrm{Id}-\mathcal{R}_{0}^{\mathrm{eo}}\right), \mathcal{R}_{1}^{\mathrm{eo}}\right]\right)=\operatorname{Ind}\left(\check{\partial}_{X_{1}}^{\mathrm{eo}}, \mathcal{R}_{1}^{\mathrm{eo}}\right) .
$$

as desired.

As a special case we consider $X_{01}=Y_{0} \times[0,1]$. In this case the formula can be rewritten as:

$$
\operatorname{Ind}\left(\check{\partial}_{X_{01}}^{\mathrm{eo}},\left[\left(\operatorname{Id}-\mathcal{R}_{0}^{\mathrm{eo}}\right), \mathcal{R}_{1}^{\mathrm{eo}}\right]\right)=\operatorname{Ind}\left(\check{\partial}_{X_{1}}^{\mathrm{eo}}, \mathcal{R}_{1}^{\mathrm{eo}}\right)-\operatorname{Ind}\left(\check{\partial}_{X_{0}}^{\mathrm{eo}}, \mathcal{R}_{0}^{\mathrm{eo}}\right) .
$$


Since $X_{1}$ is homotopic, as a $\operatorname{Spin}_{\mathbb{C}}$-manifold to $X_{0}$, we can consider $\mathcal{R}_{1}$ as defining a boundary condition on $X_{0}$. The index of $\left(\check{\beth}_{X_{1}}^{\mathrm{eo}}, \mathcal{R}_{1}^{\mathrm{eo}}\right)$ does not change as we smoothly deform $X_{1}$ to $X_{0}$, and we can therefore apply the Agranovich-Dynin formula, Theorem 8 from [9], to prove:

Corollary 5. If $Y$ is a strictly pseudoconvex, contact manifold, bounding a $\operatorname{Spin}_{\mathbb{C}}$-manifold, and $\mathcal{S}_{0}, \mathcal{S}_{1}$ are generalized Szegö projectors defined on $Y$, then

$$
\operatorname{R}-\operatorname{Ind}\left(\mathcal{S}_{0}, \mathcal{S}_{1}\right)=\operatorname{Ind}\left(\check{\partial}_{Y \times[0,1]}^{\mathrm{e}},\left[\left(\operatorname{Id}-\mathcal{R}_{0}^{\mathrm{e}}\right), \mathcal{R}_{1}^{\mathrm{e}}\right]\right) .
$$

Remark 6. This result is strongly suggested by the analysis in [9], but does not follow directly from it. It is unclear whether the result remains true if $Y$ is not the boundary of $\operatorname{Spin}_{\mathbb{C}}$-manifold.

Applying Theorem 7 twice we easily obtain a cocycle formula for these indices.

Corollary 6. Suppose that $X_{01}, X_{12}$ are $\operatorname{Spin}_{\mathbb{C}}$-manifolds with boundaries $Y_{0} \amalg Y_{1}, Y_{1} \amalg Y_{2}$, respectively. Assume that $\bar{Y}_{0}$ is also the pseudoconvex boundary of a compact $\operatorname{Spin}_{\mathbb{C}}$-manifold. Let $\mathcal{S}_{0}, \mathcal{S}_{1}, \mathcal{S}_{2}$, denote generalized Szegö projectors defined on $Y_{0}, Y_{1}, Y_{2}$, and $\mathcal{R}_{0}, \mathcal{R}_{1}, \mathcal{R}_{2}$ the modified pseudoconvex $\bar{\partial}$-Neumann boundary conditions they define. The following cocycle relation holds:

$$
\begin{aligned}
& \operatorname{Ind}\left(\check{\not}_{X_{02}}^{\mathrm{eo}},\left[\left(\mathrm{Id}-\mathcal{R}_{0}^{\mathrm{eo}}\right), \mathcal{R}_{2}^{\mathrm{eo}}\right]\right)= \\
& \operatorname{Ind}\left(\check{\partial}_{X_{01}}^{\mathrm{eo}},\left[\left(\mathrm{Id}-\mathcal{R}_{0}^{\mathrm{eo}}\right), \mathcal{R}_{1}^{\mathrm{eo}}\right]\right)+\operatorname{Ind}\left(\check{\partial}_{X_{12}}^{\mathrm{eo}},\left[\left(\mathrm{Id}-\mathcal{R}_{1}^{\mathrm{eo}}\right), \mathcal{R}_{2}^{\mathrm{eo}}\right]\right) \text {. }
\end{aligned}
$$

Remark 7. As suggested to the author by Laszlo Lempert, one might try to extend the notion of the relative index between pairs of generalized Szegö projectors defined on one contact manifold, to a relative index between pairs of generalized Szegő projectors defined on pairs of "almost complex $\operatorname{Spin}_{\mathbb{C}^{-}}$ cobordant" contact manifolds, $\left(Y_{0}, H_{0}\right),\left(Y_{1}, H_{1}\right)$. By this we mean that there is a $\operatorname{Spin}_{\mathbb{C}}$-manifold with boundary $X_{01}$ such that $b X_{01}=Y_{1} \amalg \bar{Y}_{0}$, and the

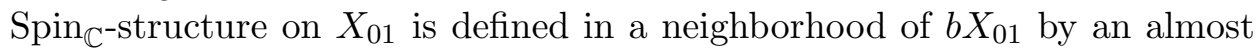
complex structure. The almost complex structure induces the given contact structures on the boundary components, and the boundary components are strictly pseudoconvex, resp. pseudoconcave.

Let $\mathcal{S}_{0}, \mathcal{S}_{1}$ be generalized Szegő projectors defined on $\left(Y_{0}, H_{0}\right),\left(Y_{1}, H_{1}\right)$, respectively. Generalizing (89), one might attempt to define

$$
" \mathrm{R}-\operatorname{Ind}\left(\mathcal{S}_{0}, \mathcal{S}_{1}\right) "=\operatorname{Ind}\left(\check{\mathrm{e}}_{X_{01}}^{\mathrm{e}},\left[\left(\operatorname{Id}-\mathcal{R}_{0}^{\mathrm{e}}\right), \mathcal{R}_{1}^{\mathrm{e}}\right]\right) .
$$


Corollary 6 shows that this invariant satisfies the cocycle formula. The problem with this definition is that it seems unlikely that two different choices of

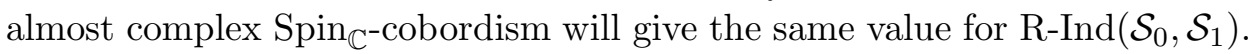
If $X_{01}^{\prime}$ is another such cobordism, then this amounts to knowing whether or not $\operatorname{Ind}\left(\check{\partial}_{X_{01}}^{\mathrm{e}} \amalg \bar{X}_{01}^{\prime}\right)$ vanishes.

While this definition does not appear to be adequate, it seems likely that one could modify the definition in (91) by subtracting a topological or geometric invariant of the cobordism, $\mathcal{T}\left(X_{01}\right)$, with the properties that

$$
\begin{aligned}
\mathcal{T}\left(X_{01}\right)+\mathcal{T}\left(\bar{X}_{01}^{\prime}\right) & =\operatorname{Ind}\left(\varlimsup_{X_{01} \amalg \bar{X}_{01}^{\prime}}\right) \\
\mathcal{T}(Y \times[0,1]) & =0 .
\end{aligned}
$$

The modified invariant would then agree with $\mathrm{R}-\operatorname{Ind}\left(\mathcal{S}_{0}, \mathcal{S}_{1}\right)$ in the product case, and would depend only on the pair $\left(Y_{0}, H_{0}, \mathcal{S}_{0}\right),\left(Y_{1}, H_{1}, \mathcal{S}_{1}\right)$.

In our earliest work on relative indices between classical Szegő projectors we had a variety of conditions assuring that R-Ind $\left(\mathcal{S}_{0}, \mathcal{S}_{1}\right)$ vanishes, see [5]. Following the philosophy of the remark, we have a considerable generalization of our earlier results.

Theorem 8. Let $X$ be a strictly pseudoconvex, complex manifold with boundary, on which there is defined an exhaustion function, $\varphi$. For each $c \in \mathbb{R}$ let

$$
X_{c}=\varphi^{-1}((-\infty, c]) \text { and } X^{c}=X \backslash X_{c} .
$$

Suppose that for some $c_{0}, \varphi$ is strictly plurisubharmonic in $X^{c_{0}}$. For $c>c_{0}$, a regular value of $\varphi$, let $\mathcal{S}_{0}$ be the classical Szegö projector defined on $b X_{c}$, $\mathcal{S}_{1}$, the classical Szegö projector defined on bX, and $\mathcal{R}_{0}, \mathcal{R}_{1}$, the modified pseudoconvex $\bar{\partial}$-Neumann boundary conditions they define. Under these assumptions

$$
\operatorname{Ind}\left(\check{\partial}_{X^{c}}^{\mathrm{e}},\left[\left(\mathrm{Id}-\mathcal{R}_{0}^{\mathrm{e}}\right), \mathcal{R}_{1}^{\mathrm{e}}\right]\right)=0 .
$$

Proof. The gluing formula (83) implies that (94) is equivalent to the statement that

$$
\operatorname{Ind}\left(\varpi_{X}^{\mathrm{e}}, \mathcal{R}_{1}^{\mathrm{e}}\right)=\operatorname{Ind}\left(\varpi_{X^{c}}^{\mathrm{e}}, \mathcal{R}_{0}^{\mathrm{e}}\right) .
$$

Since we are working in the integrable case we can apply equation 77 of [7] to conclude that

$$
\begin{aligned}
& \operatorname{Ind}\left(\check{\partial}_{X}^{\mathrm{e}}, \mathcal{R}_{1}^{\mathrm{e}}\right)=\sum_{q=1}^{n}(-1)^{q} \operatorname{dim} H^{0, q}(X) \\
& \operatorname{Ind}\left(\check{\partial}_{X^{c}}^{\mathrm{e}}, \mathcal{R}_{0}^{\mathrm{e}}\right)=\sum_{q=1}^{n}(-1)^{q} \operatorname{dim} H^{0, q}\left(X_{c}\right),
\end{aligned}
$$


where $n=\operatorname{dim}_{\mathbb{C}} X$. As there is a strictly plurisubharmonic exhaustion defined in $X^{c},\left(X, X_{c}\right)$ is a Runge pair. Hence, we can apply the classical results of Andreotti, Grauert and Hörmander to conclude that

$$
H^{0, q}(X) \simeq H^{0, q}\left(X_{c}\right) \text { for } 1 \leq q \leq n .
$$

See [16]. The theorem follows immediately from (96) and (97).

\section{Sub-elliptic boundary conditions along a sepa- rating hypersurface}

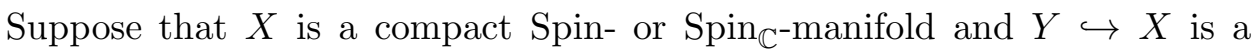
separating hypersurface; let $X \backslash Y=X_{0} \amalg X_{1}$. Let $\mathcal{P}_{0}, \mathcal{P}_{1}$ be Calderon projectors defined on $X_{0}, X_{1}$ respectively. Bojarski's theorem expresses the $\operatorname{Ind}\left(\partial_{X}\right)$ as the relative index:

$$
\operatorname{Ind}\left(\check{\partial}_{X}^{\mathrm{e}}\right)=\mathrm{R}-\operatorname{Ind}\left(\mathcal{P}_{1}^{\mathrm{e}},\left(\operatorname{Id}-\mathcal{P}_{0}^{\mathrm{e}}\right)\right) .
$$

If $P$ is a classical pseudodifferential projector acting on $\$ \uparrow Y$, so that $P \mathcal{P}_{0}+$ $(\mathrm{Id}-P)\left(\mathrm{Id}-\mathcal{P}_{0}\right)$ is classically elliptic, then $\left(\check{\partial}_{X_{0}}^{\mathrm{e}}, P^{\mathrm{e}}\right)$ and $\left(\check{\partial}_{X_{1}}^{\mathrm{e}},\left(\mathrm{Id}-P^{\mathrm{e}}\right)\right)$ are Fredholm operators. Expressing the indices of these operators as relative indices, and using the cocycle relation for relative indices, Bojarski's theorem easily implies that

$$
\operatorname{Ind}\left(\widetilde{\partial}_{X}^{\mathrm{e}}\right)=\operatorname{Ind}\left(\widetilde{\partial}_{X_{0}}^{\mathrm{e}}, P^{\mathrm{e}}\right)+\operatorname{Ind}\left(\widetilde{\partial}_{X_{1}}^{\mathrm{e}},\left(\operatorname{Id}-P^{\mathrm{e}}\right)\right)
$$

In [7] we generalized this identity to the sub-elliptic case, but only under

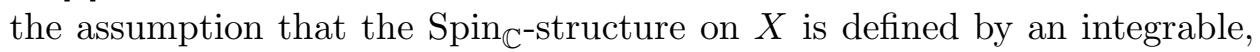
almost complex structure. In this section we use the relative index formalism developed here and in [9] to extend this formula to the general case.

Theorem 9. Let $X$ be a $\operatorname{Spin}_{\mathbb{C}}$-manifold and $Y \hookrightarrow X$, a separating hypersurface; let $X \backslash Y=X_{0} \amalg X_{1}$. Suppose that the $\operatorname{Spin}_{\mathbb{C}}$-structure is defined in a neighborhood of $Y$ by an almost complex structure, inducing a contact structure on $Y=Y_{1} \amalg \cdots \amalg Y_{N}$ with definite Levi-form. We suppose that the components $Y_{1}, \ldots, Y_{L}$ are strictly pseudoconcave, and $Y_{L+1}, \ldots, Y_{N}$ are strictly pseudoconvex, with respect to $X_{0}$. For each boundary component we choose a generalized Szegö projector, $\left\{\mathcal{S}_{i}: i=1, \ldots, N\right\}$, and let $\mathcal{R}_{0}$ be the modified $\bar{\partial}$-Neumann boundary condition they define as in (61), then

$$
\operatorname{Ind}\left(\check{\partial}_{X}^{\mathrm{e}}\right)=\operatorname{Ind}\left(\check{\partial}_{X_{0}}^{\mathrm{e}}, \mathcal{R}_{0}^{\mathrm{e}}\right)+\operatorname{Ind}\left(\mathrm{\partial}_{X_{1}}^{\mathrm{e}},\left(\operatorname{Id}-\mathcal{R}_{0}^{\mathrm{e}}\right)\right) \text {. }
$$


Proof. Let $\mathcal{P}_{0}$ and $\mathcal{P}_{1}$ denote Calderon projectors defined, using the invertible doubles $X_{0} \amalg \bar{X}_{0}$, and $X_{1} \amalg \bar{X}_{1}$, on $b X_{0}$ and $b X_{1}$, respectively The indices on the right hand side of (100) can be computed, using Theorem 5 , as relative indices:

$$
\begin{aligned}
\operatorname{Ind}\left({ }_{X_{0}}^{\mathrm{e}}, \mathcal{R}_{0}^{\mathrm{e}}\right) & =\operatorname{R}-\operatorname{Ind}\left(\mathcal{P}_{0}^{\mathrm{e}}, \mathcal{R}_{0}^{\mathrm{e}}\right) \\
\operatorname{Ind}\left({ }_{X_{1}}^{\mathrm{e}},\left(\operatorname{Id}-\mathcal{R}_{0}^{\mathrm{e}}\right)\right) & =\mathrm{R}-\operatorname{Ind}\left(\mathcal{P}_{1}^{\mathrm{e}},\left(\operatorname{Id}-\mathcal{R}_{0}^{\mathrm{e}}\right)\right) .
\end{aligned}
$$

Corollary 4 applies to show that

$$
\operatorname{R-Ind}\left(\mathcal{P}_{1}^{\mathrm{e}},\left(\operatorname{Id}-\mathcal{R}_{0}^{\mathrm{e}}\right)\right)=-\mathrm{R}-\operatorname{Ind}\left(\left(\mathrm{Id}-\mathcal{P}_{1}^{\mathrm{e}}\right), \mathcal{R}_{0}^{\mathrm{e}}\right) .
$$

We are left to show that:

$$
\operatorname{R}-\operatorname{Ind}\left(\mathcal{P}_{0}^{\mathrm{e}},\left(\operatorname{Id}-\mathcal{P}_{1}^{\mathrm{e}}\right)\right)=\mathrm{R}-\operatorname{Ind}\left(\mathcal{P}_{0}^{\mathrm{e}}, \mathcal{R}_{0}^{\mathrm{e}}\right)-\mathrm{R}-\operatorname{Ind}\left(\left(\operatorname{Id}-\mathcal{P}_{1}^{\mathrm{e}}\right), \mathcal{R}_{0}^{\mathrm{e}}\right)
$$

The result then follows from Bojarski's theorem. The proof of (103) is essentially identical to the proof of Proposition 13 in [9]. The difference here is that in our earlier paper $X_{0}$ and $X_{1}$ are both pseudoconvex, so we worked with $X_{0}$ and $\bar{X}_{1}$. This is why $\left(\mathrm{Id}-\mathcal{P}_{1}^{\mathrm{e}}\right)$ appears in the second term of (103), instead of $\mathcal{P}_{1}^{\mathrm{e}}$, as in equation (204) of [7]. The argument in [9] relies on general properties of the parametrix $\mathcal{U}$ and indices of tame Fredholm pairs, which are unconnected to the number, or convexity properties of the boundary components. The routine modifications needed to establish (103) are left to the reader.

\section{The non-separating case}

Not yet considered is the case of a non-separating hypersurface $Y$ in a compact $\operatorname{Spin}_{\mathbb{C}^{-}}$manifold. We make our usual assumptions regarding the $\operatorname{Spin}_{\mathbb{C}^{-}}$ structure on $X$ : the structure is induced, in a neighborhood of $Y$ by an almost complex structure. The almost complex structure defines a contact structure on $Y$, with respect to which the Levi-form is definite. The manifold with boundary $X_{01}=X \backslash Y$, has two boundary components, $Y_{0}, Y_{1}$, both isomorphic to $Y$. For simplicity we limit ourselves to the case that $Y$ is connected, though the results proved here clearly extend to the case that $Y$ has several components.

Following our practice above, we label the components so that $Y_{1}$ is a strictly pseudoconvex boundary and $Y_{0}$, a strictly pseudoconcave boundary. Let $\mathcal{S}$ be a generalized Szegö projector defined on $Y$, and $\mathcal{R}$ the modified pseudoconvex $\bar{\partial}$-Neumann boundary operator it defines. The boundary 


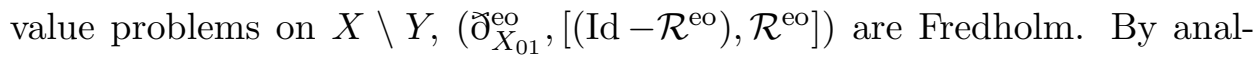
ogy to the previous results we would expect that the index of this operator computes the index of the closed manifold,

$$
\operatorname{Ind}\left(\check{\partial}_{X}^{\mathrm{eo}}\right)=\operatorname{Ind}\left(\check{\partial}_{X_{01}}^{\mathrm{eo}},\left[\left(\mathrm{Id}-\mathcal{R}^{\mathrm{eo}}\right), \mathcal{R}^{\mathrm{eo}}\right]\right) .
$$

To prove this we use a device suggested by [4: We attach a collar $\overline{Y \times[0,1]}$, to the boundary of $X_{01}$. To do this we first flatten the $\operatorname{Spin}_{\mathbb{C}^{-}}$ structure in a neighborhood of $b X_{01}$. This does not change $\operatorname{Ind}\left(\check{\partial}_{X_{01}}^{\mathrm{eo}},\left[\left(\mathrm{Id}-\mathcal{R}^{\mathrm{eo}}\right), \mathcal{R}^{\mathrm{eo}}\right]\right)$, and

$$
\operatorname{Ind}\left(\varpi_{X}\right)=\operatorname{Ind}\left(\varpi_{X_{01} \amalg \overline{Y \times[0,1]}}\right) .
$$

Thus Theorem 9 implies the following formula for the index of $\not_{X}^{\mathrm{eo}}$ :

$$
\begin{aligned}
\operatorname{Ind}\left(\check{\partial}_{X}^{\mathrm{eo}}\right)=\operatorname{Ind}\left(\check{\partial}_{X_{01}}^{\mathrm{eo}},\left[\left(\operatorname{Id}-\mathcal{R}^{\mathrm{eo}}\right), \mathcal{R}^{\mathrm{eo}}\right]\right)- & \\
& \operatorname{Ind}\left(\check{\partial}_{Y \times[0,1]}^{\mathrm{eo}},\left[\left(\operatorname{Id}-\mathcal{R}^{\mathrm{eo}}\right), \mathcal{R}^{\mathrm{eo}}\right]\right) .
\end{aligned}
$$

We are therefore reduced to showing that

$$
\operatorname{Ind}\left(\mathrm{\partial}_{Y \times[0,1]}^{\mathrm{eo}},\left[\left(\mathrm{Id}-\mathcal{R}^{\mathrm{eo}}\right), \mathcal{R}^{\mathrm{eo}}\right]\right)=0 .
$$

This can easily be established by a direct calculation.

Let $\theta$ be a one-form defining the contact structure $H$ on $Y$ and $J$ a complex structure on the fibers of $H$ so that $\mathcal{L}_{J}=d \theta(\cdot, J \cdot)$ is positive definite on $H \times H$. $T$ denotes the Reeb vector field: $\theta(T)=1, i_{T} d \theta=0$. We use $\mathcal{L}_{J}$ to define the metric on $H$ and declare $T$ to be orthogonal to $H$ and of unit length. With this data the $\operatorname{Spin}_{\mathbb{C}}$-bundle on $Y$ satisfies

$$
\$_{Y} \simeq \bigoplus_{q=0}^{n-1} \Lambda_{b}^{0, q} Y .
$$

We realize $\Lambda_{b}^{0,1} Y$ as a subbundle of $T^{*} Y \otimes \mathbb{C}$ by requiring the restriction to $T_{b}^{1,0} Y \oplus\{\mathbb{C} T\}$ to vanish. Let $\rho$ denote a coordinate on $[0,1]$. We extend the almost complex structure to $Y \times[0,1]$ be defining $J \partial_{\rho}=T$, and the metric, by declaring $\partial_{\rho}$ to have unit length, and to be orthogonal to $T Y$.

The spin-bundle on $Y \times[0,1]$ is isomorphic to $\$_{Y \times[0,1]}=\bigoplus \Lambda^{0, q}(Y \times$ $[0,1])$, with the obvious splitting into even and odd forms. Clearly $\$_{Y}$, pulled back to $Y \times[0,1]$, is canonically a subbundle of $\$_{Y \times[0,1]}$ under these identifications. We can write a section of $\$_{Y \times[0,1]}$ in the form

$$
\sigma=\sigma_{t}(\rho)+\bar{\partial} \rho \wedge \sigma_{n}(\rho)
$$


where $\sigma_{t}(\rho), \sigma_{n}(\rho)$ are 1-parameter families of sections of $\$ \$_{Y}$, that is elements of $\mathcal{C}^{\infty}\left([0,1] ; \mathcal{C}^{\infty}\left(\$_{Y}\right)\right)$. If $\sigma$ is a section of $\$_{Y \times[0,1]}^{\mathrm{e}}$, then $\sigma_{t}$ is a 1 parameter family of even-degree sections of $\$_{Y}$, and $\sigma_{n}$ is a 1 parameter family of odddegree sections of $\$_{Y}$. Analogous statements are true for sections of $\$_{Y \times[0,1]}^{\circ}$. The isomorphism of $\$_{Y \times[0,1]}^{\mathrm{e}}$ with $\$_{Y}$ just takes $\sigma^{\mathrm{e}} \rightarrow \sigma_{t}+\sigma_{n}$.

Under this identification the operator $\mathrm{\partial}_{Y \times[0,1]}^{\mathrm{e}}$ becomes

$$
\mathrm{\partial}_{Y \times[0,1]}^{\mathrm{e}} \leftrightarrow \partial_{\rho}+B
$$

where $B$ is the self-adjoint Dirac-operator on $Y$. As $\mathcal{L}_{J}$ is positive definite, the end $Y \times\{1\}$ is strictly pseudoconvex and $Y \times\{1\}$ is strictly pseudoconcave. The boundary condition $\left[\left(\mathrm{Id}-\mathcal{R}^{\mathrm{e}}\right), \mathcal{R}^{\mathrm{e}}\right]$ becomes:

$$
\begin{array}{rlrl}
\mathcal{S} \sigma_{t}^{0,0}(1) & =0 & \sigma_{n}(1)=0 \\
(\operatorname{Id}-\mathcal{S}) \sigma_{t}^{0,0}(0) & =0 & & \sigma_{t}(0)=0 .
\end{array}
$$

The odd-part $\left(\check{\partial}_{Y \times[0,1]}^{\mathrm{o}},\left[\left(\mathrm{Id}-\mathcal{R}^{\mathrm{o}}\right), \mathcal{R}^{\mathrm{o}}\right]\right)$ is the adjoint of $\left(\partial_{Y \times[0,1]}^{\mathrm{e}},\left[\left(\mathrm{Id}-\mathcal{R}^{\mathrm{e}}\right), \mathcal{R}^{\mathrm{e}}\right]\right)$ and so, under these identifications, we have

$$
\mathrm{\partial}_{Y \times[0,1]}^{\mathrm{o}} \leftrightarrow-\partial_{\rho}+B
$$

and the boundary condition, $\left[\left(\operatorname{Id}-\mathcal{R}^{\circ}\right), \mathcal{R}^{\circ}\right]$ becomes:

$$
\begin{aligned}
(\mathrm{Id}-\mathcal{S}) \sigma_{t}^{0,0}(1) & =0 & & \sigma_{t}(1)=0 \\
\mathcal{S} \sigma_{t}^{0,0}(0) & =0 & & \sigma_{n}(0)=0 .
\end{aligned}
$$

With these preliminaries, it is now easy to see that the kernel and cokernel of $\left(\widetilde{\partial}_{Y \times[0,1]}^{\mathrm{e}},\left[\left(\mathrm{Id}-\mathcal{R}^{\mathrm{e}}\right), \mathcal{R}^{\mathrm{e}}\right]\right)$ are isomorphic and therefore:

$$
\operatorname{Ind}\left(\check{\partial}_{Y \times[0,1]}^{\mathrm{e}},\left[\left(\mathrm{Id}-\mathcal{R}^{\mathrm{e}}\right), \mathcal{R}^{\mathrm{e}}\right]\right)=0 .
$$

Suppose that $\sigma(\rho) \in \mathcal{C}^{\infty}\left([0,1] ; \mathcal{C}^{\infty}\left(\$_{Y}\right)\right)$ represents an element of the nullspace of this operator. Clearly $\tilde{\sigma}(\rho)=\sigma(1-\rho)$, then belongs to the null-space of $\left(\check{\partial}_{Y \times[0,1]}^{\mathrm{o}},\left[\left(\mathrm{Id}-\mathcal{R}^{\mathrm{o}}\right), \mathcal{R}^{\mathrm{o}}\right]\right)$. As this is the adjoint operator, the assertion of (114) follows immediately.

This completes the proof of the following theorem:

Theorem 10. Let $X$ be a compact $\operatorname{Spin}_{\mathbb{C}}$-manifold and $Y \hookrightarrow X$, a nonseparating hypersurface. Suppose that the $\operatorname{Spin}_{\mathbb{C}}$-structure is induced, in a neighborhood of $Y$ by an almost complex structure, with respect to which $Y$ is a contact manifold with a definite Levi-form. Let $X_{01}=X \backslash Y$, and $\mathcal{S}$ be a generalized Szego" projector defined on $Y$, with $\mathcal{R}$ the modified pseudoconvex $\bar{\partial}-$ Neumann boundary operator it defines. We have that:

$$
\operatorname{Ind}\left(\check{\partial}_{X}^{\mathrm{eo}}\right)=\operatorname{Ind}\left(\check{\partial}_{X_{01}}^{\mathrm{eo}},\left[\left(\mathrm{Id}-\mathcal{R}^{\mathrm{eo}}\right), \mathcal{R}^{\mathrm{eo}}\right]\right) .
$$




\section{Stein fillings for 3-manifolds}

We now show how to use the gluing results for the relative index to prove our main result, Theorem 1. For this result we assume that $(Y, H)$ is a compact 3-dimensional, contact manifold with a strictly pseudoconvex CRstructure, $T_{b}^{0,1} Y$, supported by $H$, that arises as the boundary of a strictly pseudoconvex complex manifold, $X_{+}$. Let $\mathcal{S}_{0}$ denote the classical Szegő projector onto boundary values of holomorphic functions defined on $X_{+}$. In addition we assume that $\left(Y, T_{b}^{0,1} Y\right)$ arises as the pseudoconcave boundary of a smooth complex manifold with boundary $X_{-}$, and that $X_{-}$contains a positive, smooth, compact holomorphic curve, $Z$. By positive we mean that there is a strictly plurisubharmonic exhaustion function, $\varphi$ defined in $X_{-} \backslash Z$, so that $b X_{-}=\varphi^{-1}(0)$, and $\varphi(x)$ tends to infinity as $x \rightarrow Z$. We extend $\varphi$ smoothly to $X_{+}$so that $X_{+}=\varphi^{-1}((-\infty, 0])$. For $c \in \mathbb{R}$, we let

$$
X_{c}=\varphi^{-1}((-\infty, c]) \text {. }
$$

Proof of Theorem 11. The hypothesis of the theorem includes the requirement that $H_{c}^{2}\left(X_{-} ; \Theta\right)=0$. The basic result of Kiremidjian implies that any sufficiently small perturbation, $\omega$, of the CR-structure on $b X_{-}$can be extended to define an integrable deformation, $\Omega$, of the complex structure on $X_{-}$. If we choose a sufficiently large $c \in \mathbb{R}$, then $Y_{c}=\varphi^{-1}(c)$ is the strictly pseudoconcave boundary of small tubular neighborhood of $Z$. The manifold $Y_{c}$ is diffeomorphic to a circle bundle in the normal bundle to $Z$, $N Z=T^{1,0} X_{-} \uparrow_{Z} / T^{1,0} Z$. Indeed, it is not difficult to show that the contact structure on $Y_{c}$ is isotopic to the standard $U(1)$-invariant contact structure on the unit circle in $N Z$ defined by a metric on $N Z$ with positive curvature.

The $\bar{\partial}$-operator defined by the deformed complex structure, $\bar{\partial}_{\Omega}$ satisfies:

$$
\bar{\partial}_{\Omega}=\bar{\partial}_{0}+P_{\Omega}
$$

where $P_{\Omega}$ is a first order operator with smooth coefficients bounded in the $\mathcal{C}^{1}$-topology by $C\|\Omega\|_{\mathcal{C}^{k}}$, for some $C \in \mathbb{R}, k \in \mathbb{N}$. Using the Banach space version of Kiremidjian's theorem proved in [10], it follows that for another $C^{\prime}, k^{\prime}$ these coefficients are bounded in the $\mathcal{C}^{1}$-topology by $C^{\prime}\|\omega\|_{\mathcal{C}^{k^{\prime}}}$. If we fix a $c \in \mathbb{R}$, as above, then, provided that $\|\omega\|_{\mathcal{C}^{k^{\prime}}}$ is sufficiently small, the exhaustion function $\varphi$ remains strictly plurisubharmonic, with respect to $\bar{\partial}_{\Omega}$, on $X_{c} \cap X_{-}$.

Now suppose that the deformed CR-structure on $Y$ is fillable, and so it can also be realized as the boundary of strictly pseudoconvex complex manifold, $X_{+}^{\prime}$. We let $X^{\prime}=X_{+}^{\prime} \amalg X_{-}^{\prime}$, where $X_{-}^{\prime}$ denotes $X_{-}$with the 
deformed complex structure defined by $\Omega$.. Let $\mathcal{S}_{1}$ denote the Szegö projector onto the boundary values of holomorphic functions defined on $X_{+}^{\prime}, \widetilde{\mathcal{S}}_{0}$ the Szegö projector on $b X_{c}$ with respect to the original complex structure, and $\widetilde{\mathcal{S}}_{1}$ the Szegö projector on $b X_{c}^{\prime}$, with respect to the deformed complex structure. To prove the theorem we show that

$$
\operatorname{R-Ind}\left(\mathcal{S}_{0}, \mathcal{S}_{1}\right)=\operatorname{R}-\operatorname{Ind}\left(\widetilde{\mathcal{S}}_{0}, \widetilde{\mathcal{S}}_{1}\right) .
$$

From the hypothesis we know that $\operatorname{deg} N Z \geq 2 g-1$, where $g$ is the genus of $Z$. Thus $b X_{c}$ is covered by the Theorem of Stipsicz: Amongst Stein fillings of a circle bundle of degree $d$ over a surface with genus $g$, with the standard contact structure, if $d \geq 2 g-1$, then the signature and Euler characteristic are bounded, see [18]. Using the formula from [9]:

$$
\begin{aligned}
\operatorname{R}-\operatorname{Ind}\left(\widetilde{\mathcal{S}}_{0}, \widetilde{\mathcal{S}}_{1}\right)=\operatorname{dim} H^{0,1}\left(X_{c}\right) & -\operatorname{dim} H^{0,1}\left(X_{c}^{\prime}\right)+ \\
& \frac{\operatorname{sig}\left(X_{c}\right)-\operatorname{sig}\left(X_{c}^{\prime}\right)+\chi\left(X_{c}\right)-\chi\left(X_{c}^{\prime}\right)}{4},
\end{aligned}
$$

we conclude that $\mathrm{R}$ - $\operatorname{Ind}\left(\widetilde{\mathcal{S}}_{0}, \widetilde{\mathcal{S}}_{1}\right)$ assumes only finitely many values. Note that the Stipsicz result has no smallness assumption on the size of the perturbation of the CR-structure.

We let $\mathcal{R}_{0}, \mathcal{R}_{1}, \widetilde{\mathcal{R}}_{0}, \widetilde{\mathcal{R}}_{1}$ denote the modified pseudoconvex $\bar{\partial}$-Neumann boundary conditions defined by these Szegö projectors. Because we have strictly plurisubharmonic exhaustion functions defined on the collars,

$$
X_{c-}=X_{c} \cap X_{-} \text {and } X_{c-}^{\prime}=X_{c}^{\prime} \cap X_{-}^{\prime},
$$

we can apply Theorem 8 to conclude that

$$
\begin{aligned}
& \operatorname{Ind}\left(\varpi_{X_{c}}, \widetilde{\mathcal{R}}_{0}\right)=\operatorname{Ind}\left(\varlimsup_{X_{+}}, \mathcal{R}_{0}\right) \\
& \operatorname{Ind}\left(\varpi_{X_{c}^{\prime}}, \widetilde{\mathcal{R}}_{1}\right)=\operatorname{Ind}\left(\varpi_{X_{+}^{\prime}}, \mathcal{R}_{1}\right) .
\end{aligned}
$$

We can add a collars to both pairs, $X_{c}, \bar{X}_{c}^{\prime}$ and $X_{+}, \bar{X}_{+}^{\prime}$, to obtain com-

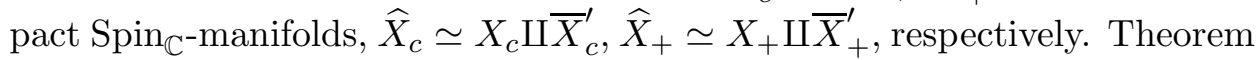
9 of [9] applies to show that

$$
\begin{aligned}
& \operatorname{R}-\operatorname{Ind}\left(\mathcal{S}_{0}, \mathcal{S}_{1}\right)=\operatorname{Ind}\left(\check{\partial}_{\widehat{X}_{+}}^{\mathrm{e}}\right)-\operatorname{Ind}\left(\varlimsup_{X_{+}}, \mathcal{R}_{0}\right)+\operatorname{Ind}\left(\varlimsup_{X_{+}^{\prime}}, \mathcal{R}_{1}\right), \\
& \operatorname{R-Ind}\left(\widetilde{\mathcal{S}}_{0}, \widetilde{\mathcal{S}}_{1}\right)=\operatorname{Ind}\left(\widetilde{\partial}_{\widehat{X}_{c}}^{\mathrm{e}}\right)-\operatorname{Ind}\left(\widetilde{\partial}_{X_{c}}, \widetilde{\mathcal{R}}_{0}\right)+\operatorname{Ind}\left(\varlimsup_{X_{c}^{\prime}}, \widetilde{\mathcal{R}}_{1}\right) .
\end{aligned}
$$

Combining these formulæ with those in (121) we see that

$$
\operatorname{R-Ind}\left(\mathcal{S}_{0}, \mathcal{S}_{1}\right)-\operatorname{R-Ind}\left(\widetilde{\mathcal{S}}_{0}, \widetilde{\mathcal{S}}_{1}\right)=\operatorname{Ind}\left(\widetilde{\varpi}_{\widehat{X}_{+}}^{\mathrm{e}}\right)-\operatorname{Ind}\left(\widetilde{\partial}_{\widehat{X}_{c}}^{\mathrm{e}}\right)
$$




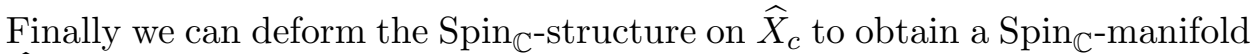

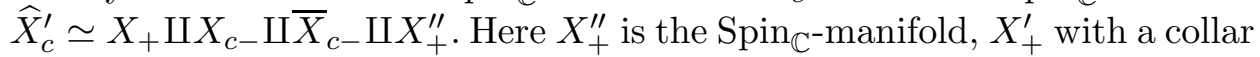

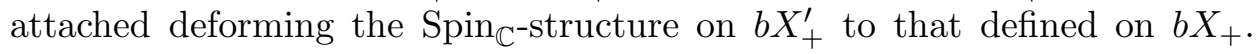
Clearly this deformation does not change $\operatorname{Ind}\left(\widetilde{\partial}_{\widehat{X}_{c}}^{\mathrm{e}}\right)$, moreover $X_{+} \amalg X_{+}^{\prime \prime} \simeq$ $\widehat{X}_{+}$.

The excision theorem of Gromov and Lawson (see Chapter 10 of [2]) applies to show that

$$
\begin{aligned}
\operatorname{Ind}\left(\varlimsup_{\widehat{X}_{c}^{\prime}}^{\mathrm{e}}\right) & =\operatorname{Ind}\left(\check{\partial}_{X_{+} \amalg X_{+}^{\prime \prime}}^{\mathrm{e}}\right)+\operatorname{Ind}\left(\check{\partial}_{X_{c-} \amalg \bar{X}_{c-}}^{\mathrm{e}}\right) \\
& =\operatorname{Ind}\left(\widetilde{\partial}_{\widehat{X}_{+}}^{\mathrm{e}}\right) .
\end{aligned}
$$

The second term vanishes because $X_{c-} \amalg \bar{X}_{c-}$ is an invertible double. This completes the proof of (118), and thereby the proof of the theorem.

One might reasonably enquire when the geometric hypotheses in equation (18) hold. A simple case to consider is that of line bundle over a Riemann surface, $L \rightarrow \Sigma$. Let $g$ denote the genus of $\Sigma$ and $d=\operatorname{deg} L$. In [11] we compute $H_{c}^{2}\left(X_{;} \Theta\right)$, where $X_{-}$is a neighborhood of the zero section in $L$. We use the $S^{1}$-action to decompose $H_{c}^{2}\left(X_{-} ; \Theta\right)$ into Fourier components:

$$
H_{c}^{2}\left(X_{-} ; \Theta\right) \simeq \bigoplus_{k=-1}^{\infty} H_{c}^{2}\left(X_{-} ; \Theta\right)_{(k)}
$$

With $\kappa$ the canonical bundle of $\Sigma$, the Fourier components fit into long exact sequences:

$$
\left[H_{c}^{2}\left(X_{-} ; \Theta\right)_{(-1)}\right]^{\prime} \simeq H^{0}\left(\Sigma ; \kappa^{2} \otimes L^{-1}\right)
$$

for $k \geq 0$ :

$$
\begin{aligned}
H^{0}\left(\Sigma ; \kappa \otimes L^{-k-2}\right) & \longrightarrow\left[H_{c}^{2}\left(X_{-} ; \Theta\right)_{(k)}\right]^{\prime} \\
H^{0}\left(\Sigma ; \kappa^{2} \otimes L^{-k-2}\right) & \longrightarrow H^{0}\left(\Sigma ; L^{k+2}\right) \longrightarrow \cdots
\end{aligned}
$$

If $\operatorname{deg} L \geq 3 g-3$, then $\operatorname{deg} \kappa^{2} \otimes L^{-1} \leq g-1$, and generically

$$
\left[H_{c}^{2}\left(X_{-} ; \Theta\right)_{(-1)}\right]^{\prime} \simeq H^{0}\left(\Sigma ; \kappa^{2} \otimes L^{-1}\right)=0
$$

see [13]. The other Fourier components are easily seen to vanish. This improves upon our earlier result where we proved a similar bound on the relative index assuming that $d>4 g-3$. This proves the following: 
Proposition 7. Suppose that $L \rightarrow \Sigma$ is a line bundle over a surface, with $\operatorname{deg} L$ at least $3 g-3$, where $g$ is the genus of $\Sigma$. Let $\widetilde{L}$ denote the compactification of $L$ obtained by adding the "section at $\infty$." For generic complex structures on $L$ and $\Sigma$, the set of small embeddable perturbations of the $C R$ structure on a strictly pseudoconvex hypersurface, $Y \subset \widetilde{L}$, such that the zero section of $L$ lies in the pseudoconcave component of $\widetilde{L} \backslash Y$, is closed in the $\mathcal{C}^{\infty}$-topology.

Proof. The hypersurface $Y$ bounds a strictly pseudoconcave domain, $X_{-}$, in $\widetilde{L}$, which contains the zero section. The genericity assumption implies that the cohomology group $H_{c}^{2}\left(X_{-} ; \Theta\right)$ vanishes. Hence we can apply Theorem 1 to conclude that the relative index between the Szegö projector on $Y$, and any small embeddable perturbation is uniformly bounded. Using Theorem E in [5] we complete the proof of the Proposition.

Remark 8. This result generalizes Lempert's Theorem 1.1 from 17, covering strictly pseudoconvex hypersurfaces in $\mathbb{C}^{2} \subset \mathbb{P}^{2}$, in that the hypersurface is not assumed to be the boundary of a tubular neighborhood of the zero section of $L$. For boundaries of small tubular neighborhoods we have a stronger result: the set of all embeddable perturbations is closed in the $\mathcal{C}^{\infty}$-topology provided that $\operatorname{deg} L>2 g-2$, see [9]. In the latter case there is no smallness hypothesis.

\section{References}

[1] R. Beals And P. Greiner, Calculus on Heisenberg Manifolds, vol. 119 of Annals of Mathematics Studies, Princeton University Press, 1988.

[2] B. Booss-Bavnbek and K. P. Wojciechowsi, Elliptic Boundary Problems for the Dirac Operator, Birkhäuser, Boston, 1996.

[3] L. Boutet de Monvel and V. Guillemin, The spectral theory of Toeplitz operators, vol. 99 of Ann. of Math. Studies, Princeton University Press, 1981.

[4] X. Dai and W. Zhang, Splitting of the family index, Comm. Math. Phys., 182 (1996), pp. 303-318.

[5] C. L. Epstein, A relative index on the space of embeddable CRstructures, I, II, Annals of Math., 147 (1998), pp. 1-59, 61-91. 
[6] _ Geometric bounds on the relative index, Jour. Inst. Math. Jussieu, 1 (2002), pp. 441-465.

[7] —, Subelliptic Spin $\mathbb{C}$ Dirac operators,I, to appear Annals of Math., (2006), pp. 1-36.

[8] - Subelliptic Spin $\mathbb{C}$ Dirac operators, II, to appear Annals of Math., (2006), pp. $1-54$.

[9] — Subelliptic Spin $\mathbb{C}$ Dirac operators, III, to appear Annals of Math., (2006), pp. 1-72.

[10] C. L. Epstein And G. M. Henkin, Extension of CR-structures for 3-dimensional pseudoconcave manifolds, in Multidimensional Complex Analysis and Partial Differential Equations, São Carlos (1995), vol. 205 of Contemp. Math, Providence, RI, 1997, Amer. Math. Soc., pp. 51-67.

[11] — Stability of embeddings for pseudoconcave surfaces and their boundaries, Acta Math., 185 (2000), pp. 161-237.

[12] C. L. Epstein And R. Melrose, Contact degree and the index of Fourier integral operators, Math. Res. Letters, 5 (1998), pp. 363-381.

[13] P. Griffiths And J. Harris, Principles of Algebraic Geometry, Wiley-Interscience, NewYork, 1978.

[14] M. Gromov and H. Lawson, Positive scalar curvature and the Dirac operator on complete Riemannian manifolds, Publ. Math. IHES, 58 (1983), pp. 295-408.

[15] V. Guillemin, V. Ginzburg, and Y. Karshon, Moment maps, cobordisms, and Hamiltonian group actions, vol. 98 of Mathematical Surveys and Monographs, American Mathematical Society, Providence, RI, 2002.

[16] L. HöRmANDER, $l_{2}$ estimates and existence theorems for the $\bar{\partial}$ operator, Acta Math., 113 (1965), pp. 89-152.

[17] L. LEMPERT, Embeddings of three dimensional Cauchy-Riemann manifolds, Math. Ann., 300 (1994), pp. 1-15.

[18] A. I. Stipsicz, On the geography of Stein fillings of certain 3-manifolds, Michigan Math. J., 51 (2003), pp. 327-337. 
[19] M. E. TAYLOR, Noncommutative microlocal analysis, part I, vol. 313 of Mem. Amer. Math. Soc., AMS, 1984. 